\title{
Cortical and thalamic connectivity of posterior parietal visual cortical areas PPc and PPr of the domestic ferret (Mustela putorius furo).
}

\author{
Leigh-Anne Dell $^{1}$, Giorgio M. Innocenti ${ }^{2,3}$, Claus C. Hilgetag ${ }^{1,4}$ and Paul R. Manger ${ }^{5}$
}

1 Institute of Computational Neuroscience, University Medical Center Hamburg-Eppendorf, Germany

2 Department of Neuroscience, Karolinska Institute, Stockholm, Sweden

3 Brain and Mind Institute, École Polytechnique Fédérale de Lausanne, Switzerland

4 Department of Health Sciences, Boston University, USA

5 School of Anatomical Sciences, Faculty of Health Sciences, University of the Witwatersrand, Johannesburg, South Africa

Grant Sponsor: The work was supported by the Swedish Medical Research Foundation (No. 12594. G.M.I.), a Wenner-Gren Foundation fellowship (P.R.M), DFG projects SFB 936/A1 (L.A.D. and C.C.H.) and SFB 926/ Z3 and TRR 169/A2 (C.C.H.).

${ }^{*}$ Correspondence to: Paul Manger; School of Anatomical Sciences, Faculty of Health Sciences, University of the Witwatersrand, 7 York Road, Parktown, 2193, Johannesburg, Republic of South Africa. Paul.Manger@wits.ac.za;

Ph: +27117172497

Fax: +27117172422 . 


\begin{abstract}
:
The present study describes the ipsilateral and contralateral cortico-cortical and cortico-thalamic connectivity of the parietal visual areas PPc and PPr in the ferret using standard anatomical tract-tracing methods. The two divisions of posterior parietal cortex of the ferret are strongly interconnected, however area PPc shows stronger connectivity with the occipital and suprasylvian visual cortex, while area PPr shows stronger connectivity with the somatomotor cortex, reflecting the functional specificity of these two areas. This pattern of connectivity is mirrored in the contralateral callosal connections. In addition, PPc and PPr are connected with the visual and somatomotor nuclei of the dorsal thalamus. Numerous connectional similarities exist between the posterior parietal cortex of the ferret (PPc and PPr) and the cat (area 7 and 5 ), indicative of the homology of these areas within the Carnivora. These findings highlight the existence of a fronto-parietal network as a shared feature of the organization of parietal cortex across Euarchontoglires and Laurasiatherians, with the degree of expression varying in relation to the expansion and areal complexity of the posterior parietal cortex. This observation indicates that the ferret is a potentially valuable experimental model animal for understanding the evolution and function of the posterior parietal cortex and the fronto-parietal network across mammals. The data generated will also contribute to the Ferretome (www.ferretome.org) connectomics databank, to further cross-species analyses of connectomes and illuminate wiring principles of cortical connectivity across mammals.
\end{abstract}

Keywords: Mustela putorius furo; hodology; cortical evolution; Carnivora; corpus callosum; fronto-parietal network. 


\section{Introduction}

The posterior parietal cortex is a multisensory cortical territory, of varying size within and between different mammalian lineages, situated between the unimodal somatosensory and visual cortical territories (e.g. Hyvärinen, 1982; Caminiti, Innocenti, \& Battaglia-Mayer, 2015; Kaas \& Stepniewska, 2016; Goldring \& Krubitzer, 2017; Kaas, Qi, \& Stepniewska, 2017). This region of cortex is involved in many functions including sensory perception, motor activity and behavioural modulation (see Hyvärinen, 1982 for an extensive review). Studies of the parietal region of the cortex have mostly been undertaken in primates, due to the expansion of this region in the primate lineage and especially so in humans (Hill, Inder, Neil, Diesker, Harwell, \& Van Essen, 2010), the importance of this region in visuallyguided motor tasks (Creem-Regehr, 2009), and the small size of this cortical region in other members of the Euarchontoglires (Kolb \& Walkey, 1987; Wallace, Ramachandran, \& Stein, 2004; Remple, Reed, Stepniewska, \& Kaas, 2006; Remple, Reed, Stepniewska, Lyon, \& Kaas, 2007; Goldring \& Krubitzer, 2017). In primates, and especially in humans, this cortical territory is thought to be parcellated into many different functional regions or areas (e.g. Seltzer \& Pandya, 1980; Lewis \& Van Essen, 2000a,b; Nelson et al., 2010; Mars, Sallet, Schüffelgen, Jbabdi, Toni, \& Rushworth, 2011; Stepniewska, Friedman, Gharbawie, Cerkevich, Roe, \& Kaas, 2011; Kaas, Qi, \& Stepniewska, 2017), and it is heavily interconnected with motor, premotor and other cortical regions (e.g. Felleman \& Van Essen, 1991; Bullmore \& Sporns, 2005; Cloutman \& Lambon Ralph, 2012; Kaas \& Stepniewska, 2016; Kaas et al., 2017). In the smallest-brained primate where posterior parietal cortex has been extensively studied (Galago garnetti), this cortical territory has been subdivided into three distinct areas - rostral (visuomotor), caudal (visual) and medial (possibly visuomotor) (Fang, Stepniewska, \& Kaas, 2005; Stepniewska, Fang, \& Kaas, 2009a; Stepniewska, Cerkevich, Fang, \& Kaas, 2009b; Kaas \& Stepniewska, 2016), with the rostral area being heavily interconnected with the motor and pre-motor cortex (Stepniewska et al., 2011; Kaas \& Stepniewska, 2016; Kaas et al., 2017).

In contrast to the studies undertaken in mammals belonging to the Euarchontoglires, studies of species within the other major Eutherian mammal radiation, the Laurasiatheria, are more limited, and only found in species of the order Carnivora. Brodmann (1909) provided an architectonic study of the posterior parietal cortex in the kinkajou, but the majority of studies have focused on the cat (Heath \& Jones, 1971; Marcotte \& Updyke, 1982; Symonds \& Rosenquist, 1984; Olson \& Lawler, 1987; Pigarev \& Rodionova, 1998). In addition, two studies have examined the posterior parietal cortex of the ferret (Manger, Masiello, \& Innocenti, 2002b; Homman-Ludiye, Manger, \& Bourne, 2010). These studies, through architecture, physiological mapping and limited connectional studies, have revealed the presence of two distinct cortical areas within the ferret posterior parietal cortex, a caudal unimodal visual area and a rostral bimodal somatovisual area.

The ferret posterior parietal cortex appears to be organized in a manner quite similar to that observed in the galago, although the mosaic or fractured maps of sensory surfaces found in the galago and other primates (Kaas \& Stepniewska, 2016; Kaas et al., 2017) was not observed in the ferret (Manger et al., 2002b). Despite this seemingly similar organization, it must be noted that the last common ancestor of the ferret and galago occurred 85-95 million years ago (Kaschube, Schnabel, Löwel, Coppola, White, \& Wolf, 2010; Kiel et al., 2012; Kaas et al., 2017). Given that the non-primate Euarchontoglires only have a small posterior parietal cortical region (Kaas \& Stepniewska, 2016; Goldring \& Krubitzer, 2017; Kaas et al., 2017), it is highly unlikely 
that the cortical areas in the ferret and galago can be considered homologous - the relative expansion of the posterior parietal cortex in the different lineages (primate and carnivore) being potentially independent occurrences. This observation then raises the question of whether these areas, if not directly homologous, are analogous. The extensive fronto-parietal network associated with visually guided behaviour is a clear trait of the posterior parietal cortex in the primates (Kaas \& Stepniewska, 2016; Kaas et al., 2017). If such a fronto-parietal network were to exist in the ferret, the case for analogy of these regions in the different species would be quite strong, and indeed be informative about the baseline organization of the posterior parietal cortex in early Eutherian mammals, and the potential evolutionary pathways and constraints that have led to the organization of the posterior parietal cortex in the different major Eutherian mammal lineages (Euarchontoglires and Laurasiatheria). To this end, the current study examined the corticocortical and thalamic connectivity of the posterior parietal cortical areas PPc and PPr of ferret in order to determine whether an extensive fronto-parietal network is present.

\section{Material and Methods}

\section{Surgical procedure and tracer injections}

Eight adult female ferrets (Mustela putorius furo), weighing between $600 \mathrm{~g}$ and $1000 \mathrm{~g}$, were used in this current study (four injection sites per cortical area). The experiments were conducted according to the Swedish and European Community guidelines for the care and use of animals in scientific experiments. All animals were initially anesthetized with i.m. doses of ketamine hydrochloride (Ketalar, $10 \mathrm{mg} / \mathrm{kg}$ ) and medetomidin hydrochloride (Domitor, $0.08 \mathrm{mg} / \mathrm{kg}$ ), supplemented with atropine sulphate $(0.15 \mathrm{mg} / \mathrm{kg}$ ) and placed in a stereotaxic frame. A mixture of $1 \%$ isoflurane in a 1:1 nitrous oxide and oxygen mixture was delivered through a mask while the animal maintained its own respiration. Anesthetic level was monitored using the eye blink and withdrawal reflexes, in combination with heart rate measurement. The parietal cortex was exposed under aseptic conditions and in each animal numerous (but fewer than 20) electrophysiological recordings were taken to ensure correct placement of the tracer within a specific cortical area (Manger et al., 2002b). Approximately 500 $\mathrm{nl}$ of tracer (biotinylated dextran amine, BDA $10 \mathrm{k}, 5 \%$ in $0.1 \mathrm{M}$ phosphate buffer; Molecular Probes) was delivered at each injection site using a Hamilton microsyringe (Figs. 1, 2a, 2b). After the completion of the injections, a soft contact lens was cut to fit over the exposed cortex, while the retracted dura mater was pulled over the contact lens and the excised portion of bone repositioned and held in place with dental acrylic. The temporal muscle was reattached using surgical glue and the midline incision of the skin sutured. Antibiotics were administered (Terramycin, $40 \mathrm{mg} / \mathrm{kg}$, daily for 5 days) and these animals were given a 2-week recovery period to allow for tracer transport. At the end of this period, the animals were euthanized with a lethal dose of sodium pentobarbital (80 mg/kg, i.p.) and perfused intracardially, initially with a rinse of $0.9 \%$ saline $\left(4^{\circ} \mathrm{C}, 500 \mathrm{ml} / \mathrm{kg}\right)$, followed by fixation with $4 \%$ paraformaldehyde in $0.1 \mathrm{M}$ phosphate buffer $\left(4^{\circ} \mathrm{C}, 1000 \mathrm{ml} / \mathrm{kg}\right)$.

\section{Sectioning and staining procedures}

The brains were removed from the skull and post-fixed overnight in $4 \%$ paraformaldehyde in $0.1 \mathrm{M}$ phosphate buffer and then transferred to a $30 \%$ sucrose solution in $0.1 \mathrm{M}$ phosphate buffer $\left(4^{\circ} \mathrm{C}\right)$ and allowed to equilibrate. The brains were either: (1) frozen in dry ice and sectioned at $50 \mu \mathrm{m}$ on a freezing microtome in a coronal plane $(4$ cases, two for each of the parietal cortical 
areas, one in the medial and one in the lateral portion of each area) for a one in four series for Nissl (cresyl violet), myelin (Gallyas, 1979), cytochrome oxidase (Carroll \& Wong-Riley, 1984) and BDA; or (2) cryoprotected and the cerebral cortex dissected away from the remainder of the brain and the dorsolateral surface semi-flattened (4 cases, two for each of the parietal cortical areas, one in the medial and one in the lateral portion of each area) between two glass slides, frozen onto the cold microtome stage and sectioned parallel to the semi-flattened surface at 50 $\mu \mathrm{m}$ for a one in two series for BDA and cytochrome oxidase. For BDA tracer visualization, the sections were incubated in $0.5 \%$ bovine serum albumin in $0.05 \mathrm{M}$ Tris buffer for $1 \mathrm{~h}$, followed by incubation in an avidin-HRP solution for $3 \mathrm{~h}$. A 10 min pre-incubation in $0.2 \% \mathrm{NiNH}_{4} \mathrm{SO}_{4}$ preceded the addition of $\mathrm{H}_{2} \mathrm{O}_{2}(200 \mu \mathrm{l} / \mathrm{l})$ to this solution, at which time the sections were monitored visually for the reaction product. To stop the reaction, the sections were placed in $0.05 \mathrm{M}$ Tris buffer. All sections were mounted on $0.5 \%$ gelatine coated slides, dehydrated in a graded series of alcohols, cleared in xylene and coverslipped with Depex mounting medium. All injection sites resulted in robust anterograde and retrograde transport of tracer in both the cerebral cortex (Fig. 2c-f) and the visual and motor portions of the dorsal thalamus (Fig. 3).

\section{Qualitative and quantitative analysis}

For qualitative analysis, the stained sections were examined under low and high power magnification using a light microscope to determine in which sections through the cortex labelled cell bodies and terminals were present. Under low power stereomicroscopy using the flattened sections, the edges of each section were drawn with the aid of a camera lucida, and the location of the injection site marked. Areal borders were delineated and drawn using the cytochrome oxidase stained sections. The sections reacted for BDA were then matched to these drawings and the locations of the individual retrogradely labelled cells plotted and regions of anterogradely labelled axonal terminals demarcated. The drawings were scanned and redrawn using a Canvas $X$ Pro 16 drawing program (ACD Systems International Inc., USA). Digital photomicrographs were captured using a Zeiss Axioskop and the Axiovision software. No pixilation adjustments or manipulation of the captured images were undertaken, except for contrast and brightness adjustment using Adobe Photoshop 7.

To quantify the retrograde BDA labelling per injection site, and control for variance in the size of the injection, a fraction of labelled neurons ( $N \%$, Table 1) was calculated using the formula:

$\mathrm{N} \%=\Sigma$ Projection neurons identified within a region $/ \Sigma$ Total projection neurons identified across both hemispheres $\times 100$

Where cell bodies could be clearly identified, neurons within the injection halo were counted. Furthermore, cell bodies located on boundary lines between areas were only accounted for in the region where the most overlap was present. 


\section{Abbreviations}

3a, rostral, or proprioceptive, somatosensory area

17, primary visual cortex

18 , second visual cortical area

19, third visual cortical area

20a, temporal visual area a

20b, temporal visual area $b$

21, fourth visual cortical area

A, A lamina of the lateral geniculate nucleus

A1, A1 lamina of the lateral geniculate nucleus

Al, primary auditory cortex

AAF, anterior auditory field

ADF, anterior dorsal auditory field

AEV, anterior ectosylvian visual area

AMLS, anteromedial lateral suprasylvian visual area

ALLS, anterolateral lateral suprasylvian visual area

AUD, auditory cortex

AV, anterior ventral nucleus of the dorsal thalamus

AVF, anterior ventral auditory field

C, C lamina of the lateral geniculate nucleus

DLS, dorsal lateral suprasylvian visual cortical area

is, injection site

LP, lateral posterior nucleus of the dorsal thalamus

Is, lateral sulcus

M1, primary motor cortex

MIN, medial intralaminar nucleus of the lateral geniculate nucleus

OB, olfactory bulb

OT, olfactory tract

$\mathbf{P}$, perigeniculate lamina of the lateral geniculate nucleus

PIR, piriform cortex

PMLS, posteromedial lateral suprasylvian visual area

PLLS, posterolateral lateral suprasylvian visual area

Po, posterior nucleus of the dorsal thalamus

PPc, posterior parietal caudal cortical area

PPr, posterior parietal rostral cortical area

PreF, prefrontal cortical region

PreM, premotor cortical region

PS, posterior suprasylvian visual cortical area

PSF?, potential posterior suprasylvian auditory field

Pul, pulvinar nucleus of the dorsal thalamus

SI, primary somatosensory area

SII/PV, second somatosensory area/parietoventral somatosensory area

SIII, third somatosensory area

SMA?, potential supplementary motor area

VA, ventral anterior nucleus of the dorsal thalamus

Vb, ventrobasal complex of the dorsal thalamus

VLS, ventral lateral suprasylvian visual area 
bioRxiv preprint doi: https://doi.org/10.1101/491993; this version posted December 10, 2018. The copyright holder for this preprint (which was not certified by peer review) is the author/funder. All rights reserved. No reuse allowed without permission.

VL, ventral lateral nucleus of the dorsal thalamus VP?, ventral posterior ectosylvian region. 


\section{Results}

In the current study, anatomical tract tracer injections were placed into the medial and lateral aspects of the caudal and rostral posterior parietal cortical areas (PPr and PPc) of the ferret, in order to examine the cortico-cortical and cortico-thalamic connections of these two parietal cortical areas. Despite minor quantitative differences being observed between the injections placed in the lateral and medial aspects of both PPc and PPr, the overall distribution of labelled connections was identical between the lateral and medial portions of each cortical area. Thus, the description of connectivity provided herein has grouped all four injections in each area for brevity.

It was evident in all cases that there was more connectivity in the hemisphere ipsilateral to the injection site than in the contralateral hemisphere, and that this label was clustered in its distribution (Table 1, Fig. 4). In all cases, ipsilateral connectivity was strongest within the parietal visual areas, the somatosensory areas, and the suprasylvian visual area AMLS. Moderate connectivity strength was observed the occipital visual areas and the remaining suprasylvian visual areas, while weak connectivity was observed in the temporal visual areas and frontal cortex. Contralateral connectivity was strongest in the regions homotopic to the injection site, while connectivity with the dorsal thalamus was found primarily in specific visual and motor nuclei.

\section{Connectivity of PPC}

Injection of tracer into PPc revealed widespread connectivity throughout the vast majority of the ipsilateral cerebral cortex (Fig. 5), with connections observed in parietal, occipital, suprasylvian and temporal visual regions, throughout the somatomotor and prefrontal regions, the auditory and multimodal cortex of the anterior and middle ectosylvian gyrus, as well as with the cortex overlying the claustrum. The only region of the cerebral cortex devoid of any connectivity was the posterior ectosylvian gyrus (Fig. 5). Within area PPc, extensive and dense reciprocal connectivity was observed throughout the entire cortical area. This extensive reciprocal connectivity was also observed throughout area PPr, although if, for example, the injection site in PPc was located in the lateral portion of this area, the reciprocal connectivity in the medial region of PPr was weaker than that observed in the lateral region (Fig. 5). Within areas 17 and 18, patches of weak anterograde projections were observed, and while these patches sometimes contained retrogradely labeled cells, the distribution of anterograde and retrograde label was mostly independent of each other.

In contrast, in areas 19 and 21, the patches of anterograde label were larger and covered the majority of each of these cortical areas, and these patches often contained moderate densities of retrogradely labeled cells. The density of labeling in areas $\mathbf{1 9}$ and $\mathbf{2 1}$ was always stronger in their more medial aspects, which lie closer to the injection sites, but to reiterate, connectivity was observed through both cortical areas (Fig. 5). Of the suprasylvian visual regions, strong reciprocal connectivity was observed throughout area AMLS and the medial half of PMLS, with weaker reciprocal connectivity in the lateral half of PMLS. Moderately dense reciprocal connectivity was observed throughout area ALLS, while weak reciprocal connectivity was observed in area PLLS, with areas DLS and VLS only having weak retrograde connectivity (Fig. 5). Within the visual temporal areas, weak reciprocal connectivity was observed in areas 20 a and $\mathbf{2 0 b}$, with area PS being devoid of connections. 
All somatosensory cortical areas exhibited reciprocal connections with area PPc. The densest reciprocal connectivity was observed in area SIII, with patches of connectivity found throughout the area, but as with area PPr, if the injection site in PPc was located in the lateral portion of the area, the connectivity in area SIII was strongest in the lateral portion and weaker in the medial portion (Fig. 5). Areas SII, PV, SI and 3a all exhibited patches of weak anterograde connectivity throughout much of their extent, and these patches were often coincident with patches of retrogradely labeled neurons, although often the anterograde and retrograde connections were not matching. In the primary motor (M1) and premotor regions, patches of anterograde projections, with occasional retrogradely labeled neurons were observed, with the anterograde projections occupying much of the mediolateral extent of the premotor cortical region (Fig. 5). Similarly, weak patches of anterograde projections, with occasional retrogradely labeled cells were observed in the lateral half of the orbital gyrus, presumably representing the prefrontal cortex. As mentioned, connectivity was observed in the anterior and middle portions of the ectosylvian gyrus, but not the posterior portion of this gyrus. In the middle ectosylvian gyrus, weak reciprocal connectivity was observed in the primary auditory cortex (A1), the anterior auditory field (AAF) and the anterior dorsal (ADF) and anterior ventral (AVF) auditory fields. Similarly, weak reciprocal connectivity was observed in the multimodal anterior ectosylvian visual area (AEV), with patches of weak anterograde label being observed rostral to this area in the cortex overlying the claustrum. The strongest ipsilateral retrograde connectivity was observed within PPc (N\% = 25.93\%), with a decrease in connectivity strength in area $\mathbf{2 1}(\mathrm{N} \%=$ $16.92 \%)$, followed by areas $18(\mathrm{~N} \%=9.78 \%), \operatorname{PPr}(\mathrm{N} \%=9.55 \%), 19(\mathrm{~N} \%=6.93 \%)$, AMLS $(\mathrm{N} \%$ $=6.70 \%)$ and PMLS (N\% = 4.38\%), with minor connections in other cortical areas (Fig. 4, Table 1).

Contralateral cortico-cortical connectivity following tracer injection in PPc was substantially weaker and less diffuse than that observed in the ipsilateral hemisphere (Fig. 5). In the contralateral PPc, a moderately dense cluster of reciprocal connectivity was found homotopic to the injection site, and this cluster spread throughout much of the contralateral PPc; that is, if the injection was placed in the lateral portion of PPc, label was also observed in the contralateral medial portion of PPc, albeit much weaker than in the homotopic lateral region of PPc (Fig. 5). Moderately dense reciprocal connectivity was also observed throughout the contralateral PPr, but more restricted in its mediolateral extent than seen for PPc. Within the contralateral suprasylvian areas, weak reciprocal connectivity was observed in areas AMLS and PMLS, while weak anterograde connectivity was observed in ALLS and PLLS, with a few retrogradely labeled cells being observed in the contralateral VLS (Fig. 5). Patches of weak reciprocal connectivity were observed in occipital areas 18, 19 and 21, but no label was observed in area 17. A small patch of weak anterograde connectivity was observed at the border of temporal areas 20a and 20b. Occasional retrogradely labeled neurons were observed surrounding these patches of reciprocal connectivity in these three occipital areas. Weak patches of reciprocal connectivity were observed in the somatosensory areas SIII and SII, with the extent of the anterograde projections being larger than those of the retrograde connections (Fig. 5). Weak reciprocal connectivity was also observed in the multimodal temporal area AEV, and in the anterior ventral (AVF) and anterior dorsal (ADF) fields of auditory cortex, with a small patch of weak anterograde label found rostral to these regions in the cortex overlying the claustrum. Thus, the connectivity between the hemispheres is far weaker than that within the hemisphere (Table 1, Figs. 4, 5), and these contralateral connections are substantially less widespread than the ipsilateral connections following injections of tracer into PPc. 
Following injection of tracer into PPc, widespread, mostly reciprocal, connectivity was observed in specific nuclei of the visual and motor portions of the dorsal thalamus (Figs. 3a, 6). Within the lateral geniculate nucleus, no anterograde labeling was observed, but retrogradely labeled neurons were seen sparsely distributed throughout lamina A/A1, lamina $\mathbf{C}$ and in the MIN. Thus, while the lateral geniculate nucleus does project to PPc, it does not receive projections from this cortical area. Both the lateral posterior and pulvinar visual thalamic nuclei were reciprocally connected with area PPc (Fig. 6). In both nuclei large, often dense, patches of anterograde label were observed throughout the rostrocaudal extent of these nuclei. Within these patches of anterograde label a moderate density of retrogradely labeled cells were also observed. In addition, low densities of retrogradely labeled cells were observed beyond these patches of reciprocal connectivity. At more rostral levels, substantial patches of reciprocal connectivity were observed throughout the ventral anterior (VA) nucleus, part of the motor system, and as with the connectivity of the lateral posterior and pulvinar nuclei, low densities of retrogradely labeled cells were found surrounding these patches of reciprocal connectivity (Fig. 6a-c). Thus, PPc appears to be most strongly reciprocally connected with the lateral posterior, pulvinar (visual) and VA (motor) nuclei of the dorsal thalamus.

\section{Connectivity of PPr}

As with area PPc, injection of tracer into PPr revealed widespread connectivity throughout the vast majority of the ipsilateral cerebral cortex (Fig. 7). The only regions of the cerebral cortex where connectivity was sparse were areas 17, 18, and the middle and posterior portions of the ectosylvian gyrus (Fig. 7). Within area PPr, extensive and high density reciprocal connectivity was observed throughout this cortical area, but if, for example, the injection was made in the medial portion of PPr, the strongest connectivity was observed in the medial portion, with somewhat weaker connectivity observed in the lateral portion (Fig. 7). Strong reciprocal connectivity was observed throughout area PPc following tracer injection into area PPr, with again the differential between medial and lateral portion connectivity strength based on the location of the injection site. Within area 17 only the occasional weak patch of anterograde projections was observed, while in area 18 slightly more weak patches of anterograde projections, associated with a small number of retrogradely labeled cells, was observed. The extent of the connectivity increased progressively in areas 19 and $\mathbf{2 1}$, with the majority of area 21 showing weak to moderate reciprocal connectivity with area PPr (Fig. 7). Within the suprasylvian visual areas, weak to moderate reciprocal connectivity was observed throughout areas AMLS and ALLS, with areas PMLS and PLLS showing similar connectivity in the medial half of these areas. A weak patch of anterograde projections was observed in area DLS, while area VLS was devoid of any connections to PPr. Of the temporal visual areas, both areas 20a and PS showed no connections with PPr, and a weak patch of anterograde projections was observed in area 20b and in the ventral posterior ectosylvian region (VP), this being the only region of the posterior ectosylvian gyrus showing connectivity to PPr (Fig. 7).

All somatosensory cortical areas showed connectivity to area PPr, but this was very weak in area 3a. The most extensively connected somatosensory area was area SIII, which showed high to moderate densities of reciprocal connectivity throughout. Moderate to low- density reciprocal connectivity was observed in areas SI, SII/PV (Fig. 7). A large and broad weak anterograde projection was observed throughout much of the primary motor cortex (M1) and the premotor cortical region, although only a few retrogradely labeled neurons were observed in $\mathbf{M} \mathbf{1}$, and no retrogradely labeled cells were observed in the premotor region. Patches of weak anterograde projections were observed in the caudal half of the orbital gyrus, presumably the prefrontal cortex. Within the middle ectosylvian gyrus, small clusters of retrogradely labeled 
neurons were observed in the anterior auditory field (AAF), while in the anterior ectosylvian gyrus weak anterograde projections were observed in the multimodal area AEV. Rostral to the anterior ectosylvian gyrus, in the cortex overlying the claustrum, mismatched patches of weak anterograde and retrograde label was observed (Fig. 7). The strongest ipsilateral retrograde connectivity was observed within area $\operatorname{PPr}(\mathrm{N} \%=27.32 \%)$, with a decrease in connectivity strength in PPc (N\% = 19.43\%) and SIII (N\% = 17.09\%), followed by areas $21(\mathrm{~N} \%=6.92 \%)$, SI $(\mathrm{N} \%=6.20 \%)$, SII/PV (N\% = 4.09\%), AMLS (N\% = 2.62\%) and M1 (N\% = 2.59\%), with minor connections in other cortical regions (Table 1, Fig. 4).

Contralateral cortico-cortical connectivity following tracer injection in PPr was substantially weaker and less diffuse than that observed in the ipsilateral hemisphere (Fig. 7). In the contralateral PPr, moderately dense reciprocal connectivity was observed throughout this cortical area. Such reciprocal connectivity was also found throughout area PPc, although the numbers of retrogradely labelled neurons was higher in the region corresponding to the injection site, that is, if the injection was made in the medial portion of PPr, the number of retrogradely labelled neurons was higher in the medial portion of the contralateral PPc (Fig. 7). Weak reciprocal connectivity was observed in the suprasylvian visual areas AMLS and PMLS, with small patches of weak anterograde projections being observed in PLLS and DLS. Weak contralateral reciprocal connectivity was only observed in area 21 of the occipital visual areas, with weak patches of anterograde connectivity observed in the temporal visual areas 20a, 20b and AEV, as well as in the posterior suprasylvian auditory field (PSF) and the multimodal ventral posterior ectosylvian region (VP). Weak, but broad, reciprocal connectivity was observed with somatosensory area SIII, while weak patches of reciprocal connectivity were observed in areas SII and SI. Patches of weak anterograde label were observed in areas 3a, primary motor cortex (M1), premotor cortex and the orbital gyrus (presumably prefrontal cortex) (Fig. 7). Lastly, isolated patches of weak anterograde projections were observed in the cortex overlying the claustrum. Thus, while the connectivity between the hemispheres is far weaker than that within the hemisphere (Table 1, Figs. 4, 7), these contralateral connections are nearly as widespread as the ipsilateral connections following injections of tracer into PPr (Fig. 7).

Following injection of tracer into PPr, widespread, mostly reciprocal, connectivity was observed throughout specific nuclei of the visual, motor and somatosensory regions of the dorsal thalamus (Figs. 3b, 8). A small number of retrogradely labeled neurons was observed in the A/A1 and C lamina and MIN of the lateral geniculate nucleus. A small patch of reciprocal connectivity was observed in the lateral posterior nucleus, with occasional retrogradely labeled neurons found surrounding this small patch of reciprocal connectivity (Fig. 8j-I). A dense patch of reciprocal connectivity was observed throughout the rostrocaudal extent of the pulvinar nucleus, with occasional retrogradely labeled cells surrounding this patch of reciprocal connectivity. Further rostral within the thalamus, a large and dense patch of reciprocal connectivity was observed throughout the majority of the ventral anterior (VA) nucleus of the motor portion of the ferret dorsal thalamus (Fig. 8a-i), but no label was observed in the ventral lateral (VL) nucleus of this region of the thalamus. Occasional retrogradely labeled neurons were observed in the ventrobasal complex $(\mathbf{V b})$, the medial division of the posterior nucleus (Po), and the anterior ventral nucleus (AV) (Fig. 8). Thus, PPr appears to be most strongly reciprocally connected with the pulvinar (visual) and ventral anterior (VA, motor) nuclei of the dorsal thalamus. 


\section{Discussion}

The present paper examined the ipsilateral and contralateral cortico-cortical and corticothalamic connectivity of the posterior parietal cortical areas PPc and PPr in the ferret, by means of standard anatomical tract-tracing methods. The data provides an understanding of the connectivity of the posterior parietal cortex of the ferret, which will contribute to the Ferretome connectivity database (www.ferretome.org; Sukhinin, Engel, Manger, \& Hilgetag, 2016), facilitate cross-species analyses of connectomes, and provide insights into proposed principles of cortical connectivity and cortical area evolution. Although PPc and PPr are strongly interconnected, it was observed that PPc was more strongly connected with the occipital and temporal visual cortex than PPr, while PPr was more strongly connected with the somatosensory cortex than PPc (Figs. 4, 5; Table 1), reflecting their specific functional aspects (Manger et al., 2002b). For all PPc and PPr injection cases, it was evident that the ipsilateral hemisphere was more strongly connected than the contralateral hemisphere, but that the distribution of connections in the contralateral hemisphere mirrored that of the ipsilateral hemisphere (Figs. 4, 5, 7; Table 1) similar to what has been observed in connectional studies of this region in the macaque monkey, rat and mouse (Barbas, Hilgetag, Saha, Dermon, \& Suski, 2005; Goulas, Uylings, \& Hilgetag, 2017; Swanson, Hahn, \& Sporns, 2017). Numerous connectional similarities and differences were observed between the posterior parietal cortex of the ferret and the cat, and the implications of these differences are discussed. Unfortunately, our understanding of the posterior parietal cortical region in the cat is incomplete (Heath \& Jones, 1971; Marcotte \& Updyke, 1982; Symonds \& Rosenquist, 1984; Olson \& Lawler, 1987; Pigarev \& Rodionova, 1998), but from the available studies it appears reasonable, for comparative purposes, to suggest that ferret areas PPc and PPr are homologous or analogous to areas $\mathbf{7}$ and $\mathbf{5}$ of the cat, respectively; however, we suggest this cautiously and use this comparison as a working hypothesis until confirmation or refutation is obtained.

\section{Area PPc connectivity - ferret vs cat}

Ferret area PPc displayed extensive ipsilateral cortical connectivity, being connected with all 15 visual areas previously described for the ferret (Fig. 9), although the strongest connectivity was with PPr, occipital visual areas 18, 19 and 21, and suprasylvian visual areas PMLS and AMLS. In addition, PPc of the ferret was connected with all known somatosensory and motor areas of the ferret, most strongly with areas SIII, SII/PV and SI. When compared to studies of the connectivity of area 7 of the cat, while the cat shows a similar overall connectional topography (Kawamura, 1973; Babb, Waters, \& Asanuma, 1984; Symonds \& Rosenquist, 1984; Olson \& Lawler, 1987; Avendaño, Rausell, Perez- Aguilar, \& Isorna, 1988; Cavada \& Reinoso-Suárez, 1985; Scannell, Blakemore, \& Young, 1995), the ferret PPc appears to have a far broader range of connections to other visual areas than observed in the cat (Fig. 9), the cat lacking connections to areas 17, PMLS, VLS, and AES; however, all of these connections, apart from the PPc-PMLS connection observed in the ferret, are weak connections. Thus, the differences observed may be due to methodology, although the strong PPc-PMLS connection present in the ferret, but absent in the cat, is a difference of note. Interestingly, the ipsilateral connectivity to the somatosensory cortex of ferret PPc and cat area 7 appear very similar, the one difference being the presence of a weak connection to SI in the ferret, which is absent in the cat (Fig. 9; Jones \& Powell, 1968; Scannell et al., 1995). The interhemispheric connectivity of ferret PPc was more restricted than its ipsilateral connectivity, although still quite broad. Significant callosal connections were observed with contralateral areas PPc, PPr, 18, 19, 21, PMLS, AMLS, SIII 
and SII, with weaker connections to other cortical areas, but notably absent from motor and premotor areas (Figs. 4,5). Studies of the interhemispheric connectivity of area 7 in the cat (possible homologue to area PPc in ferret) are limited, but the consensus is that the callosal connectivity is more restricted than the ipsilateral connectivity, a feature observed in the ferret as well as all mammalian species studied to date (Swanson et al., 2017). Furthermore, area 7 in the cat is similarly connected to its homotopic area as well as to the heterotopic area $\mathbf{5}$ (Garol, 1942; Heath \& Jones, 1971; Kawamura, 1973) (possible homologue to PPr in ferret), indicating that the posterior parietal visual regions in the ferret and the cat show similar patterns of callosal connectivity.

In terms of thalamic connectivity, area PPc of the ferret was reciprocally connected with the LP and pulvinar nuclei and received projections from the A, C and MIN laminae of the LGN. While the connections of cat area $\mathbf{7}$ with the LP and pulvinar are similar to that observed in ferret PPc, cat area $\mathbf{7}$ was reciprocally connected with lamina $\mathbf{C}$ of the LGN, but lacked connections with laminae A and MIN (Fig. 9; Heath \& Jones, 1971; Graybiel, 1972; Hendry, Jones, \& Graham, 1979; Robertson \& Cunningham, 1981; Raczkowski \& Rosenquist, 1983; Olson \& Lawler, 1987; Scannell, Burns, Hilgetag, O'Neil, \& Young, 1999). Thus, when comparing the global connectivity of ferret area PPc with cat area 7, it appears that the connectivity patterns of the ferret and cat are generally similar, but that the ferret has a more broadly connected posterior parietal unimodal visual area than the cat. While the observed differences may be methodological (e.g. different tract tracers used), there are certain differences that are unlikely to be explained methodologically, specifically the connectivity of PPc with PMLS and the various laminae of the LGN in the ferret. In our previous study of the connectivity of the occipital visual area of the ferret (Dell, Innocenti, Hilgetag, \& Manger, 2018), we noted that PMLS appears to form a hub in the network processing visual information in the ferret, and this is supported in the current study. In addition, we noted that when comparing the connectivity of the visual cortex with the LGN in the ferret and cat, that the LGN appears to be more strongly connected with visual areas early in the processing hierarchy in the cat, whereas the LGN is more strongly connected with visual areas later in the hierarchy in the ferret, and this pattern appears to continue into the posterior parietal cortex.

\section{Area PPr connectivity - ferret vs cat}

Ferret PPr, like PPc, was broadly connected with almost all ipsilateral visual cortical areas, VLS being the only area not connected with PPr (Fig. 9), but the strongest connections to other ipsilateral visual areas were with PPc, 21 and AMLS. In addition, PPr was connected with all known ipsilateral somatomotor areas of the ferret, with strongest connectivity observed with areas SIII, SI, SII/PV and M1. When compared with area $\mathbf{5}$ of the cat, the majority of these connections show strong similarity (Fig. 9; Jones \& Powell, 1968; Kawamura, 1973; Babb et al., 1984; Symonds \& Rosenquist, 1984; Avendaño et al., 1988; Cavada \& Reinoso-Suárez, 1985; Scannell et al., 1995), but there are several differences in terms of whether the connections are reciprocal, anterograde only, or retrograde only (Fig. 9). Major differences in the connectivity patterns to visual cortical areas between ferret PPr and cat area 5 include, reciprocal connections with area 17 and anterograde projections to area PS in the ferret that are absent in the cat (Fig. 9; Kawamura, 1973; Avendaño et al., 1988; Scannell et al., 1995), and mostly reciprocal connections with the suprasylvian visual areas in the ferret, whereas these are mostly retrograde connections in the cat (Fig. 9; Kawamura, 1973; Avendaño et al., 1988; Scannell et al., 1995). For connectivity with the somatomotor cortex, the areas connected in the ferret and cat appear broadly similar (Fig. 9; Jones \& Powell, 1968; Kawamura, 1973; Avendaño et al., 
1988; Scannell et al., 1995), but the ferret shows reciprocal connectivity with areas SIII, SI and 3a, whereas these regions only send connections to area $\mathbf{5}$ in the cat (Fig. 9; Jones \& Powell, 1968; Kawamura, 1973; Avendaño et al., 1988; Scannell et al., 1995). The interhemispheric connectivity of ferret PPr was more restricted than its ipsilateral connectivity, although still quite broad, especially the anterograde projections. Significant callosal connections were observed with the contralateral areas PPr, PPc and SIII, with weaker anterograde projections to many other cortical areas, including motor, premotor and prefrontal areas (Figs. 4, 7). As observed in the ferret, the interhemispheric connectivity of cat area 5 (potential homologue to ferret area PPr) was more restricted than that of the ipsilateral hemisphere and, unlike the ferret, callosal connections were observed with only areas 5 and 7 (Garol, 1942; Heath \& Jones, 1971; Kawamura, 1973), indicating that while the posterior parietal areas maintain strong interhemispheric connections in both species, these connections are broader in the ferret than the cat.

In terms of thalamic connectivity, area PPr of the ferret was reciprocally connected with the LP and pulvinar nuclei and received projections from the $\mathbf{A}$ and $\mathbf{C}$ laminae of the LGN. While the connections of cat area $\mathbf{5}$ with the LP and pulvinar are similar to that observed in ferret $\mathbf{P P r}$, cat area 5 lacked connections with the LGN (Fig. 9; Heath \& Jones, 1971; Graybiel, 1972; Hendry et al., 1979; Robertson \& Cunningham, 1981; Raczkowski \& Rosenquist, 1983; Scannell et al., 1999). Thus, as with area PPc, area PPr of the ferret appears to be more broadly connected with other cortical areas than area $\mathbf{5}$ of the cat. In addition, PPr of the ferret receives projections from the LGN, which are not observed in the cat, furthering the pattern observed, where the LGN of the ferret is more strongly connected with higher order visual areas in the ferret, but with lower order visual areas in the cat. Despite these differences, the overall connectivity pattern is reasonably consistent between species and certain differences observed may be based in different methodologies. These findings support the notion that a common connectional blue print may exist across species within an order and that differences may be due to evolutionary or functional constraints or specializations.

\section{Connectivity patterns of parietal areas of the ferret and the cat}

There is clearly a global similarity in the patterns of connectivity of posterior parietal areas PPc and PPr of the ferret and area $\mathbf{5}$ and $\mathbf{7}$ of the cat, with ipsilateral and contralateral cortical areas and also with the visual thalamus. Despite this similarity, there are differences of interest that emerge from this comparison. The ferret posterior parietal areas tend to have more reciprocal connections with other cortical areas than the cat, all of which appear to tend towards retrograde connectivity (Fig. 9), indicating that the parietal areas in the cat receive more information than they send. Thus, the ferret posterior parietal cortex may play a greater role in systems level processing and information integration than that of the cat. The second difference is the extended connectivity of the LGN to the posterior parietal cortex of the ferret, which appears to be absent in the cat (Fig. 9; Robertson \& Cunningham, 1981; Raczkowski \& Rosenquist, 1983; Scannell et al., 1999). This underscores a potential emphasis in the ferret on higher order visual stimuli detection compared to lower order visual stimulus detection emphasis in the cat, similar to that seen for the occipital visual areas (Dell et al., 2018). As discussed previously (Dell et al., 2018), these differences may relate to the larger brain size of the cat compared to the ferret, the extensive phylogenetic independence of the two species, anatomical differences related to eye position (lateral in the ferret, frontal in the cat), or their specific strategies for acquiring nutrition. Further studies of species within the two major lineages of the 
carnivores are required to determine which of these possibilities explains the observed differences.

\section{Evolution of posterior parietal cortex and the fronto-parietal network}

Due to the involvement of posterior parietal cortex in visually-guided motor tasks (CreemRegehr, 2009), and the small size of this cortical region in most non-primate mammals (Kolb \& Walkey, 1987; Wallace et al., 2004; Remple et al., 2006, 2007; Goldring \& Krubitzer, 2017; Kaas et al., 2017), studies of the posterior parietal cortex have been mostly undertaken in primates. One of the central themes to emerge from these primate studies is that the posterior parietal cortex is heavily interconnected with motor and premotor cortical areas, forming a distinct reciprocal connectivity network (the fronto-parietal network) that is thought to underlie the visually-guided digital manipulative competencies of primates (e.g. Felleman \& Van Essen, 1991; Bullmore \& Sporns, 2005; Cloutman \& Lambon Ralph, 2012; Caminiti et al., 2015; Kaas \& Stepniewska, 2016; Kaas et al., 2017), especially in humans where this region is greatly expanded (Hill et al., 2010). However, the primate studies have led to an extensive parcellation of the posterior parietal cortex (Seltzer \& Pandya, 1980; Lewis \& Van Essen, 2000a,b; Nelson et al., 2010; Mars et al., 2011; Stepniewska et al., 2011; Kaas et al., 2017), making comparisons to non-primate mammalian species, where this cortical region is not greatly expanded (Goldring \& Krubitzer, 2017), difficult. In order to meaningfully compare the organization of primate posterior parietal cortex with that found in other species, the primate with the simplest organizational scheme, the galago (Fang et al., 2005; Stepniewska et al., 2009a,b, 2011; Kaas \& Stepniewska, 2016; Kaas et al., 2017), is the best starting point. Organizational schemes forwarded for other larger brained primate species, due to their complexity, are not considered here.

The posterior parietal cortex of the galago has been subdivided into three distinct cortical areas, rostral (visuomotor, heavily connected with motor and pre-motor cortex, PPCr), caudal (visual, PPCc) and medial (somatovisual, PPCm) (Kaas et al., 2017). While the PPCm identified in the galago has no clear counterpart in non-primate mammals, as this midline region has not been explored, PPCr and PPCc of the galago may be compared with PPr and PPc of the ferret (Manger et al., 2002b) and PP and OP of the megachiropterans (Rosa, 1999). Due to the lack of distinct posterior parietal cortical areas in most mammals, related to the small size of this region (Goldring \& Krubitzer, 2017; Kaas et al., 2017), and the phylogenetic positions of the primates, carnivores and megachiropterans (Arnason, Adegoke, Gullberg, Harley, Janke, \& Kullberg, 2008; Foley, Springer, \& Teeling, 2016), it would be imprudent to assume that these cortical areas in the galago (PPCr, PPCc), ferret (PPr, PPc) and megachiropteran (PP, OP) are homologous. This indicates that expansion and arealization of the posterior parietal cortex has evolved independently at least three times in mammals; unless one takes the position that the megachiropterans are a sister group to primates (Pettigrew, 1986; Pettigrew, Jamieson, Robson, Hall, McNally, \& Cooper, 1989; Pettigrew, Maseko, \& Manger, 2008), then it is reasonable to propose homology for areas PPCc/OP and PPCr/PP in galago and megachiropterans, but not with the ferret, indicating only two currently known independent expansions of the parietal cortex. Despite the intricacies of chiropteran phylogenetic relationships, it is at least reasonable to assume that, at a minimum, expansion and arealization of the posterior parietal cortex has occurred independently in certain lineages within the Euarchontoglires (primates and/or megachiropterans) and Laurasiatheria (carnivores and/or megachiropterans). That there are broad organizational similarities, in that there is a rostral somatovisual area and caudal visual area in all species that have an expanded posterior parietal cortical region regardless of their phylogenetic affinities, indicates that the arealization of 
this region is likely related to underlying functional and connectional aspects of this cortical territory.

Indeed, even in rodents and tree shrews, where the expansion and arealization of posterior parietal cortex has not occurred, indicated by the presence of a single multimodal posterior parietal region immediately caudal to somatosensory cortex (Goldring \& Krubitzer, 2017; Kaas et al., 2017), this multimodal region is connected with the motor cortex, forming the frontoparietal network. Thus, it is reasonable to conclude that the fronto-parietal network is a shared feature of all species within the Euarchontoglires and Laurasiatheria, but varies in its degree of expression. Across species, the posterior parietal cortex forms an integral component of the dorsal visual processing stream (Goodale \& Milner, 1992; Goodale, 1998; Kaas et al., 2011). Although this processing stream was initially identified as the 'where' stream, as it deciphers spatial information about objects (Goodale \& Milner, 1992; Goodale, 1998), studies in primates proposed that this stream should rather be called the 'how' stream, as its functions extend to coordinating and controlling multisensory motor operations within a spatial attentional context (Goodale \& Milner, 1992; Burish, Stepniewska, \& Kaas, 2008; Stepniewska et al., 2009b; Kaas et al., 2011). The projections of this 'how' stream are congruent with the fronto-parietal network and thus an extension of the dorsal visual processing stream (see Goodale \& Milner, 1992; Goodale, 1998). Our studies also identify this fronto-parietal network in the ferret in line with connectivity observed in other non-primate species such as the cat (Heath \& Jones, 1971; Cavada \& Reinoso-Suárez, 1985), rat (Beckstead, 1979), guinea pig (Pritzel \& Markowitsch, 1981) and tree shrew (Remple et al., 2006, 2007), although this fronto-parietal network appears to be more limited in these non-primate species. These comparative observations indicate that the ferret is a potential experimental model to understand the evolution and function of the posterior parietal cortex and the fronto-parietal network across mammals. Further studies, using long-train intracortical microstimulation in the ferret, determining whether the ferret posterior parietal cortex shows a similar mosaic organization of movement domains embedded within the gross topographic organization of this region (Manger et al., 2002b), will further our understanding of this region, not only in the ferret, but in phylogenetically and functionally relevant senses. 
Acknowledgements: The authors wish to thank Mrs. Sonata Valentiniene for her consistently high-quality histological preparations.

Conflict of Interest: The authors declare no conflicts of interest.

Role of Authors: PRM and GI designed the study and undertook the experimental aspects of the study. LAD, CCH and PRM analyzed the material and LAD wrote the first draft of the paper, which was subsequently edited by GMI, CCH and PRM. All authors had full access to all of the data in the study and take responsibility for the integrity of the data and the accuracy of the data analysis. 


\section{References:}

Arnason, U., Adegoke, J.A., Gullberg, A., Harley, E.H., Janke, A., \& Kullberg, M. (2008). Mitogenomic relationships of placental mammals and molecular estimates of their divergences. Gene, 42, 37-51. doi: 10.1016/j.gene.2008.05.024

Avendaño, C., Rausell, E., Perez-Aguilar, D., \& Isorna, S. (1988). Organization of the association cortical afferent connections of area 5: A retrograde tracer study in the cat. Journal of Comparative Neurology, 278, 1-33. doi: 10.1002/cne.902780102

Babb, R.S., Waters, R.S., \& Asanuma, H. (1984). Corticocortical connections to the motor cortex from the posterior parietal lobe (areas $5 a, 5 b, 7)$ in the cat demonstrated by the retrograde axonal transport of horseradish peroxidase. Experimental Brain Research, 54, 476484. doi: not available

Barbas, H., Hilgetag, C.C., Saha, S., Dermon, C.R., \& Suski, J.L. (2005). Parallel organization of contralateral and ipsilateral prefrontal cortical projections in the rhesus monkey. BMC Neuroscience, 6, 32. doi: 10.1186/1471-2202-6-32

Beckstead, R.M. (1979). An autoradiographic examination of corticocortical and subcortical projections of the mediodorsal-projection (prefrontal) cortex in the rat. Journal of Comparative Neurology, 184, 43-62. doi: 10.1002/cne.901840104

Bizley, J.K., Nodal, F.R., Nelken, I., \& King, A.J. (2005). Functional organization of ferret auditory cortex. Cerebral Cortex, 15, 1637-1653. doi: 10.1093/cercor/bhi042

Brodmann, K. (1909). Localisation in the cerebral cortex, translation by L.J. Garey (1999). London: Imperial College Press.

Bullmore, E.T., \& Sporns, O. (2012). The economy of brain network organization. Nature Reviews Neuroscience, 13, 336-349. doi: 10.1038/nrn3214

Burish, M.J., Stepniewska, I., \& Kaas, J.H. (2008). Microstimulation and architectonics of frontoparietal cortex in common marmosets (Callithrix jacchus). Journal of Comparative Neurology, 507, 1151-1168. doi: 10.1002/cne.21596

Caminiti, R., Innocenti, G.M., \& Battaglia-Mayer, A. (2015). Organization and evolution of parieto-frontal processing streams in monkey and humans. Neuroscience and Biobehavioral Reviews, 56, 73-96. doi: 10.1016/j.neubiorev.2015.06.014

Carroll, E.W., \& Wong-Riley, M.T.T. (1984). Quantitative light and electron microscopic analysis of cytochrome oxidase-rich zones in the striate cortex of the squirrel monkey. Journal of Comparative Neurology, 222, 1-17. doi: 10.1002/cne.902220102

Cavada, C., \& Reinoso-Suárez, F. (1985). Topographical organization of the cortical afferent connections of the prefrontal cortex in the cat. Journal of Comparative Neurology, 242, 293324. doi: $10.1002 /$ cne. 902420302 
Cloutman, L.L., \& Lambon Ralph, M.A. (2012). Connectivity-based structural and functional parcellation of the human cortex using diffusion imaging and tractography. Frontiers in Neuroanatomy, 6, 34. doi: 10.3389/fnana.2012.00034

Creem-Regehr, S.H. (2009). Sensory-motor and cognitive functions of the human posterior parietal cortex involved in manual actions. Neurobiology of Learning and Memory, 91, 166- 171. doi: 10.1016/j.nlm.2008.10.004

Dell, L.A., Innocenti, G.M., Hilgetag, C.C., \& Manger, P.R. (2018). Cortical and thalamic connectivity of occipital visual cortical areas 17, 18, 19 and 21 of the domestic ferret (Mustela putorius furo). , under review.

Fang, P.C., Stepniewska, I., \& Kaas, J.H. (2005). Ipsilateral cortical connections of motor, premotor, frontal eye, and posterior parietal fields in a prosimian primate, Otolemur garnetti. Journal of Comparative Neurology, 490, 305-333. doi: 10.1002/cne.20665

Felleman, D.J., \& Van Essen, D.C. (1991). Distributed hierarchical processing in the primate cerebral cortex. Cerebral Cortex, 1, 1-47. doi: 10.1093/cercor/1.1.1

Foley, N.M., Springer, M.S., \& Teeling, E.C. (2016). Mammal madness: is the mammal tree of life not yet resolved? Philosophical Transactions of the Royal Society of London, Series B, Biological Sciences, 317, 20150140. doi: 10.1098/rstb.2015.0140

Gallyas, F. (1979). Silver staining of myelin by means of physical development. Neurology Research, 1, 203-209. doi: 10.1080/01616412.1979.11739553

Garol, H.W. (1942). Cortical origin and distribution of corpus callosum and anterior commissure in the cat. III. Journal of Neuropathology and Experimental Neurology, 1, 422- 429. doi: 10.1097/00005072-194210000-00007

Goldring, A.B., \& Krubitzer, L.A. (2017). Evolution of parietal cortex in mammals: from manipulation to tool use. In: J.H. Kaas (Ed.), Evolution of Nervous Systems, $2^{\text {nd }}$ Edition (pp. 259-286). Cambridge MA: Academic Press.

Goodale, M.A. (1998). Visuomotor control: Where does vision end and action begin? Current Biology, 8, R489-R491. doi: 10.1016/S0960-9822(98)70314-8

Goodale, M.A., \& Milner, A.D. (1992). Separate visual pathways for perception and action. Trends in Neuroscience, 15, 20-25. doi: 10.1016/0166-2236(92)90344-8

Goulas, A., Uylings, H.B.M., \& Hilgetag, C.C. (2017). Principles of ipsilateral and contralateral cortico-cortical connectivity in the mouse. Brain Structure and Function, 222, 1281-1295. doi: 10.1007/s00429-016-1277-y

Graybiel, A.M. (1972). Some ascending connections of the pulvinar and nucleus lateralis posterior of the thalamus in the cat. Brain Research, 44, 99-125. doi: 10.1016/00068993(72)90369-1 
Heath, C.J., \& Jones, E.G. (1971). The anatomical organization of the suprasylvian gyrus of the cat. Ergebnisse Der Anatomie Und Entwicklungsgeschichte, 45, 1-64. doi: not available

Hendry, S.H.C., Jones, E.G., \& Graham, J. (1979). Thalamic relay nuclei for cerebellar and certain related fiber systems in the cat. Journal of Comparative Neurology, 185, 679-714. doi: $10.1002 /$ cne.901850406

Hill, J., Inder, T., Neil, J., Diesker, D., Harwell, J., \& Van Essen, D. (2010). Similar patterns of cortical expansion during human development and evolution. Proceedings of the National Academy of Science USA, 107, 13135-13140. doi: 10.1073/pnas.1001229107

Homman-Ludiye, J., Manger, P.R., \& Bourne, J.A. (2010). Immunohistochemical parcellation of the ferret (Mustela putorius) visual cortex reveals substantial homology with the cat (Felis catus). Journal of Comparative Neurology, 518, 4439-4462. doi: 10.1002/cne.22465

Hyvärinen, J. (1982). Posterior parietal lobe of the primate brain. Physiological Reviews, 62, 1060-1129. doi: not available

Jones, E.G., \& Powell, T.P.S. (1968). The ipsilateral cortical connexions of the somatic sensory areas in the cat. Brain Research, 9, 71-94. doi: 10.1016/0006-8993(68)90258-8

Kaas, J.H., \& Stepniewska, I. (2016). Evolution of posterior parietal cortex and parietal- frontal networks for specific actions in primates. Journal of Comparative Neurology, 524, 595-608. doi: $10.1002 /$ cne.23838

Kaas, J.H., Gharbawie, O.A., \& Stepniewska, I. (2011). The organization and evolution of dorsal stream multisensory motor pathways in primates. Frontiers in Neuroanatomy, 5, 34. doi: 10.3389/fnana.2011.00034

Kaas, J.H., Qi, H-X., \& Stepniewska, I. (2017). Evolution of parietal-frontal networks in primates. In: J.H. Kaas (Ed.), Evolution of Nervous Systems, $2^{\text {nd }}$ Edition (pp. 287-297). Cambridge MA: Academic Press.

Kaschube, M., Schnabel, M., Löwel, S., Coppola, D.M., White, L.E., \& Wolf, F. (2010). Universality in the evolution of orientation columns in the visual cortex. Science, 330,11131116. doi: 10.1126/science. 1194869

Kawamura, K. (1973). Corticocortical fiber connections of the cat cerebrum. II. The parietal region. Brain Research, 51, 23-40. doi: 10.1016/0006-8993(73)90363-6

Kiel, W., Kaschube, M., Schnabel, M., Kisvarday, Z.F., Löwel, S., Coppola, D.M., White, L.E., \& Wolf, F. (2012). Response to the comment on "universality in the evolution of orientation columns in the visual cortex". Science, 336, 413. doi: 10.1126/science.1206416

Kolb, B., \& Walkey, J. (1987). Behavioural and anatomical studies of the posterior parietal cortex in the rat. Behavioral Brain Research, 23, 127-145. doi: 10.1016/0166- 4328(87)90050-7 
Lewis, J.W., \& Van Essen, D.C. (2000a). Mapping of architectonic subdivisions in the macaque monkey, with emphasis on parieto-occipital cortex. Journal of Comparative Neurology, 428, 79111. doi: 10.1002/1096-9861(20001204)428:1<79::AID- CNE7>3.0.CO;2-Q

Lewis, J.W., \& Van Essen, D.C. (2000b). Corticocortical connections of visual, sensorimotor, and multimodal processing areas in the parietal lobe of the macaque monkey. Journal of Comparative Neurology, 428, 112-137. doi: 10.1002/1096- 9861(20001204)428:1<112::AIDCNE8>3.0.CO;2-9

Manger, P.R., Kiper, D., Masiello, I., Murillo, L., Tettoni, L., Hunyadi, Z., \& Innocenti, G.M. (2002a). The representation of the visual field in three extrastriate areas of the ferret (Mustela putorius) and the relationship of retinotopy and field boundaries to callosal connectivity. Cerebral Cortex, 12, 423-437. doi: 10.1093/cercor/12.4.423

Manger, P.R., Masiello, I., \& Innocenti, G.M. (2002b). Areal organization of the posterior parietal cortex of the ferret (Mustela putorius). Cerebral Cortex, 12, 1280-1297. doi:

10.1093/cercor/12.12.1280

Manger, P.R., Nakamura, H., Valentiniene, S., \& Innocenti, G.M. (2004). Visual areas in the lateral temporal cortex of the ferret (Mustela putorius). Cerebral Cortex, 14, 676-689. doi: 10.1093/cercor/bhh028

Manger, P.R., Engler, G., Moll, C.K.E., \& Engel, A.K. (2005). The anterior ectosylvian visual area of the ferret: a homologue for an enigmatic visual cortical area of the cat? European of Journal Neuroscience, 22, 706-714. doi: 10.1111/j.1460-9568.2005.04246.x

Manger, P.R., Engler, G., Moll, C.K.E., \& Engel, A.K. (2008). Location, architecture, and retinotopy of the anteromedial lateral suprasylvian visual area (AMLS) of the ferret (Mustela putorius). Visual Neuroscience, 25, 27-37. doi: 10.1017/S0952523808080036

Manger, P.R., Restrepo, C.E., \& Innocenti, G.M. (2010). The superior colliculus of the ferret: cortical afferents and efferent connections to dorsal thalamus. Brain Research, 1353, 74-85. doi: 10.1016/j.brainres.2010.07.085

Marcotte, R.R., \& Updyke, B.V. (1982). Thalamocortical relations of a visual area in the lateral bank of the lateral sulcus in the cat. Society of Neuroscience Abstracts, 8, 810. doi: not available.

Mars, R.B., Sallet, J., Schüffelgen, U., Jbabdi, S., Toni, I., \& Rushworth, M.F.S. (2011). Connectivity-based subdivisions of the human right "temporoparietal junction area": evidence for different areas participating in different cortical networks. Cerebral Cortex, 22, 1894-1903. doi: $10.1093 /$ cercor/bhr268

Nelson, S.M., Cohen, A.L., Power, J.D., Wig, G.S., Miezin, F.M., Wheeler, M.E., Velanova, K., Donaldson, D.I., Phillips, J.S., Schlaggar, B.L., \& Petersen, S.E. (2010), A parcellation scheme for human left lateral parietal cortex. Neuron, 67, 156-170. doi: 10.1016/j.neuron.2010.05.025 
Olson, C.R., \& Lawler, K. (1987). Cortical and subcortical afferent connections of a posterior division of feline area 7 (area 7p). Journal of Comparative Neurology, 259, 13-30. doi: $10.1002 /$ cne. 902590103

Pettigrew, J.D. (1986), Flying primates? Megabats have the advanced pathway from eye to midbrain. Science, 231, 1304-1306. doi: 10.1126/science.3945827

Pettigrew, J.D., Jamieson, B.G.M., Robson, S.K., Hall, L.S., McNally, K.I., \& Cooper, H.M. (1989). Phylogenetic relations between microbats, megabats and primates (Mammalia: Chiroptera and Primates). Philosophical Transactions of the Royal Society of London, Series B, Biological Sciences, 325, 489-559. doi: 10.1098/rstb.1989.0102

Pettigrew, J.D., Maseko, B.C., \& Manger, P.R. (2008). Primate-like retinotectal decussation in an echolocating megabat, Rousettus aegyptiacus. Neuroscience, 153, 226-231. doi: 10.1016/j.neuroscience.2008.02.019

Pigarev, I.N., \& Rodionova, E.I. (1998). Two visual areas located in the middle suprasylvian gyrus (cytoarchitectonic field 7) of cat's cortex. Neuroscience, 85, 717-732. doi: 10.1016/S03064522(97)00642-8

Pritzel, M., \& Markowitsch, H.J. (1981). Cortico-prefrontal afferents in the guinea pig. Brain Research Bulletin, 7, 427-434. doi: 10.1016/0361-9230(81)90041-1

Raczkowski, D., \& Rosenquist, A.C. (1983). Connections of the multiple visual cortical areas with the lateral posterior-pulvinar complex and adjacent thalamic nuclei in the cat. Journal of Neuroscience, 3, 1912-1942. doi: not available

Remple, M.S., Reed, J.L., Stepniewska, I., \& Kaas, J.H. (2006). Organization of frontoparietal cortex in the tree shrew (Tupaia belangerı). I. Architecture, microelectrode maps, and corticospinal connections. Journal of Comparative Neurology, 497, 133-154. doi: 10.1002/cne.20975

Remple, M.S., Reed, J.L., Stepniewska, I., Lyon, D.C., \& Kaas, J.H. (2007). The organization of frontoparietal cortex in the tree shrew (Tupaia belangeri): II. Connectional evidence for a frontalposterior parietal network. Journal of Comparative Neurology, 501, 121-149. doi: 10.1002/cne.21226

Robertson, R.T., \& Cunningham, T.J. (1981). Organization of corticothalamic projections from parietal cortex in cat. Journal of Comparative Neurology, 199, 569-585. doi: 10.1002/cne.901990409

Rosa, M.G.P. (1999). Topographic organisation of extrastriate areas in the flying fox: implications for the evolution of mammalian visual cortex. Journal of Comparative Neurology, 411, 503-523. doi: 10.1002/(SICI)1096-9861(19990830)411:3<503::AID- CNE12>3.0.CO;2-6

Scannell, J.W., Blakemore, C., \& Young, M.P. (1995). Analysis of connectivity in the cat cerebral cortex. Journal of Neuroscience, 15, 1463-1483. doi: not available 
Scannell, J.W., Burns, G.A.P.C., Hilgetag, C.C., O’Neil, M.A., \& Young, M.P. (1999). The connectional organization of the cortico-thalamic system of the cat. Cerebral Cortex, 9, 277299. doi: $10.1093 /$ cercor/9.3.277

Seltzer, B., \& Pandya, D.N. (1980). Converging visual and somatic sensory cortical input to the intraparietal sulcus of the rhesus monkey. Brain Research, 192, 339-351. doi: 10.1016/00068993(80)90888-4

Stepniewska, I., Fang, P.C.Y., \& Kaas, J.H. (2009a). Organization of the posterior parietal cortex in galagos: I. Functional zones identified by microstimulation. Journal of Comparative Neurology, 517, 765-782. doi: 10.1002/cne.22181

Stepniewska, I., Cerkevich, C.M., Fang, P.C.Y., \& Kaas, J.H. (2009b). Organization of the posterior parietal cortex in galagos: II. Ipsilateral cortical connections of physiologically identified zones within anterior sensorimotor region. Journal of Comparative Neurology, 517, 783-807. doi: $10.1002 /$ cne.22190

Stepniewska, I., Friedman, R.M., Gharbawie, O.A., Cerkevich, C.M., Roe, A.W., \& Kaas, J.H. (2011). Optical imaging in galagos reveals parietal-frontal circuits underlying motor behavior. Proceedings of the National Academy of Science USA, 108, E725-E732. doi: 10.1073/pnas.1109925108

Sukhinin, D., Engel, A.K., Manger, P.R., \& Hilgetag, C.C. (2016). Building the ferretome. Frontiers in Neuroinformatics, 10, 16. doi: 10.3389/fninf.2016.00016

Swanson, L.W., Hahn, J.D., \& Sporns, O. (2017). Organizing principles for the cerebral cortex network of commissural and association connections. Proceedings of the National Academy of Science USA, 114, E9692- E9701. doi: 10.1073/pnas.1712928114

Symonds, L.L., \& Rosenquist, A.C. (1984). Corticocortical connections among visual areas in the cat. Journal of Comparative Neurology, 229, 1-38. doi: 10.1002/cne.902290103

Wallace, M.T., Ramachandran, R., \& Stein, B.E. (2004). A revised view of sensory cortical parcellation. Proceedings of the National Academy of Science USA, 101, 2167-2172. doi: 10.1073/pnas.0305697101 


\section{Figure Legends:}

Figure 1: Locations of the injection sites in the parietal cortical areas analyzed in the current study in relation to many of the known boundaries of visual cortical areas in a dorsolateral view of the ferret brain. Closed circles represent the injections sites where the brain was sectioned in a coronal plane. Open circles represent the injections sites where the cerebral cortex was manually semi-flattened for analysis. See list for abbreviations.

Figure 2: Photomicrographs showing examples of injection sites (a, b) and labeled cells and axons terminals (c-f) that were analyzed in the current study. (a) Injection site (is) in area PPr of a semi-flattened cerebral cortex showing the spread of tracer around the injection site, indicating the areal specificity of the injections made in the current study. (b) Injection site (is) in area PPc from a coronal section through the cerebral cortex, showing the spread of tracer and the limitation of the injection site to the cerebral cortex. Labeled cells and terminals in the medial portion of area PPr (c, medial to the lateral sulcus, Is) and SIII (d, lateral to the lateral sulcus) following transport from an injection site in area PPr. (e) High magnification image of a retrogradely labeled cell in area SII following transport from the injection site depicted in $\mathbf{a}$. (f) High magnification image of anterogradely labeled axons in area $\mathbf{2 1}$ following transport from the injection site depicted in $\mathbf{a}$. Scale bar in $\mathbf{b}=500 \mu \mathrm{m}$ and applies to $\mathbf{a}$ and $\mathbf{b}$. Scale bar in $\mathbf{d}=250$ $\mu \mathrm{m}$ and applies to $\mathbf{c}$ and $\mathbf{d}$. Scale bar in $\mathbf{f}=100 \mu \mathrm{m}$ and applies to $\mathbf{e}$ and $\mathbf{f}$. In images $\mathbf{a}, \mathbf{c}, \mathbf{d}$, and $\mathbf{f}$, the midline of the brain is to the top of the image and rostral to the left. In image $\mathbf{b}$, medial is to the right and dorsal to the top. In image $\mathbf{e}$ the pial surface has been rotated to the top of the image.

Figure 3: Photomicrographs showing examples of retrogradely labelled cells and anterogradely labelled axons in the visual and motor thalamus of the ferret following injections into the parietal visual areas PPc (a), and PPr (b). (c) High magnification image of retrogradely labelled cells in the ventrobasal complex (Vb) following transport from the injection site in area PPr. (d) High magnification image of anterogradely labelled axons in the pulvinar nucleus following transport from the injection site in area PPc. Scale bar in $\mathbf{b}=500 \mu \mathrm{m}$ and applies to $\mathbf{a}$ and $\mathbf{b}$. Scale bar in $\mathbf{d}=100 \mu \mathrm{m}$ and applies to $\mathbf{c}$ and $\mathbf{d}$. In all images dorsal is to the top and medial to the right. See list for abbreviations.

Figure 4: Graphs depicting the quantitative assessment of retrograde connectivity strength within and between cortical areas in the cerebral hemispheres ipsilateral (left column) and contralateral (right column) to the injection sites made in areas PPc (top two graphs) and PPr (bottom two graphs). The values are expressed in percentages, being the fraction of labelled neurons occurring in each cortical area. See list for abbreviations. Note that the majority of retrogradely labelled cells are found within the cortical area injected, and that both parietal areas are connected to each other, both ipsilaterally and contralaterally. 
Figure 5: Location of retrogradely labelled cortical neurons (filled circles) and anterogradely labelled axons and axon terminals (dense labelling in the darker grey shading, light labelling in the lighter grey shading) following transport from the injection site (is) located in the lateral portion of area PPc. The upper larger image represent the distribution of cells and axons in the ipsilateral semi-flattened cerebral hemisphere, while the lower smaller image represents the semi-flattened cerebral hemisphere contralateral to the injection site. Note that ipsilaterally, extensive connectivity is seen through the occipital visual areas (19 and 21), the suprasylvian visual areas (AMLS, ALLS and PMLS) as well as the third somatosensory area (SIII). In addition, broad, but weaker, reciprocal connectivity is observed in the temporal visual and auditory regions, the rostral somatosensory regions, motor, pre-motor and prefrontal regions, as well as the claustrocortical regions. The contralateral connectivity is much weaker, and less widespread, but still extensive. Areal boundaries were demarcated using alternative sections stained for cytochrome oxidase and the boundaries represent approximations based on this stain and available maps of the ferret brain (Manger, Kiper, Masiello, Murillo, Tettoni, Hunyadi, \& Innocenti, 2002a; Manger et al., 2002b; Manger, Nakamura, Valentiniene, \& Innocenti, 2004; Manger, Engler, Moll, \& Engel, 2005; Manger, Engler, Moll, \& Engel, 2008; Manger, Restrepo, \& Innocenti, 2010; Bizley, Nodal, Nelken, \& King, 2005; Homman-Ludiye et al., 2010). See list for abbreviations.

Figure 6: Diagrammatic reconstructions of the location of retrogradely labelled cells (filled circles) and anterogradely labelled axons and axon terminals (dense labelling in the darker grey shading, light labelling in the lighter grey shading) in the visual and motor thalamus of the ferret following injection of tracer into the lateral portion of the parietal visual area PPc. a represents the most rostral coronal section, with I being the most caudal. Each section is approximately $400 \mu \mathrm{m}$ apart. Note the dense reciprocal connectivity with the lateral posterior (LP), pulvinar (Pul) and ventral anterior (VA) nuclei, and limited label in the lateral geniculate nucleus. In all images dorsal (D) is to the top and medial $(\mathbf{M})$ to the right. See list for abbreviations.

Figure 7: Location of retrogradely labelled cortical neurons (filled circles) and anterogradely labelled axons and axon terminals (dense labelling in the darker grey shading, light labelling in the lighter grey shading) following transport from the injection site (is) located in the medial portion of area PPr. All conventions and abbreviations as provided in the legend to Fig. 5. Note that ipsilaterally, extensive connectivity is seen through the occipital visual areas (19 and 21), the somatosensory areas (SI, SII, SIII and PV?). In addition, broad, but weaker, connectivity is observed in the suprasylvian visual area, the motor, pre-motor and prefrontal regions, as well as the claustrocortical regions. The contralateral connectivity is much weaker, and similarly widespread. See list for abbreviations.

Figure 8: Diagrammatic reconstructions of the location of retrogradely labelled cells (filled circles) and anterogradely labelled axons and axon terminals (dense labelling in the darker grey shading, light labelling in the lighter grey shading) in the visual and motor thalamus of the ferret following injection of tracer into the medial portion of the parietal visual area PPr. a represents the most rostral coronal section, with I being the most caudal. Each section is approximately $400 \mu \mathrm{m}$ apart. Note the dense connectivity with the pulvinar nucleus of the visual thalamus and the ventral anterior (VA) nucleus of the motor thalamus, with some weak connectivity with the lateral geniculate nucleus and the ventrobasal nuclei. Conventions and abbreviations as provided in the legend to Fig. 6. 
Figure 9: Wiring diagrams depicting the connectivity of areas PPc and PPr with each other, other visual cortical areas ipsilaterally, and the visual thalamus in the ferret (a), the connections of these areas observed in the ferret but not the cat (b), and the connections of these areas overs in the cat but not the ferret (c). Each colour represents a specific cortical area (PPc - red, PPr - green), with solid lines representing reciprocal connections, short dashed lines anterograde connections only, and long dashed lines retrograde connections only. (a) Of the potential 156 connections (for each cortical area 26 potential reciprocal, anterograde only or retrograde only connections) of these two areas to the different visual cortical and thalamic regions, a total of 50 connections are found in the ferret. (b) In the ferret 32 connections not found in the cat were observed, while (c) in the cat 16 connections not found in the ferret were observed (Garol, 1942; Jones \& Powell, 1968; Heath \& Jones, 1971; Kawamura, 1973;

Robertson \& Cunningham, 1981; Raczkowski \& Rosenquist, 1983; Babb et al., 1984; Symonds \& Rosenquist, 1984; Avendaño et al., 1988; Scannell et al., 1995, 1999). 
Table 1: Average fraction of retrogradely labelled neurons (N\%), in the various visual cortical areas of the ipsilateral and contralateral hemispheres of the ferret following four separate injections of biotinylated dextran amine (BDA) into the parietal visual areas PPc and PPr. See list for abbreviations. IS - injection site

\begin{tabular}{|c|c|c|c|c|c|c|c|c|c|c|c|c|c|c|c|c|c|c|c|c|c|c|c|c|}
\hline & \multicolumn{4}{|c|}{ Occipital } & \multicolumn{4}{|c|}{ Temporal } & & \multicolumn{6}{|c|}{ Suprasylvian } & \multicolumn{2}{|c|}{ Parietal } & \multicolumn{6}{|c|}{ Somatomotor } & \multirow[b]{2}{*}{ PreF } \\
\hline IS & 17 & 18 & 19 & 21 & $20 \mathrm{a}$ & $20 \mathrm{~b}$ & PS & AEV & AUD & PMLS & PLLS & AMLS & ALLS & VLS & DLS & PPc & PPr & SIII & SII/PV & SI & $3 a$ & M1 & PreM & \\
\hline \multicolumn{25}{|c|}{ Ipsilateral connectivity } \\
\hline PPc & 0.07 & 9.78 & 6.93 & 16.92 & 0.38 & 0.21 & 0.03 & 0.50 & 1.10 & 4.38 & 0.53 & 6.70 & 2.25 & 0.23 & 0.02 & 25.93 & 9.55 & 2.54 & 1.79 & 0.58 & 0.05 & 0.01 & 0.02 & 0.05 \\
\hline PPr & 0.01 & 1.31 & 0.75 & 6.92 & 0.00 & 0.00 & 0.00 & 0.18 & 0.33 & 0.59 & 0.10 & 2.62 & 0.83 & 0.00 & 0.00 & 19.43 & 27.32 & 17.09 & 4.09 & 6.20 & 0.69 & 2.59 & 0.28 & 0.02 \\
\hline \multicolumn{25}{|c|}{ Contralateral connectivity } \\
\hline PPr & 0.00 & 0.00 & 0.00 & 0.08 & 0.00 & 0.00 & 0.00 & 0.00 & 0.02 & 0.01 & 0.00 & 0.31 & 0.00 & 0.00 & 0.00 & 1.46 & 4.86 & 1.39 & 0.15 & 0.03 & 0.02 & 0.12 & 0.00 & 0.00 \\
\hline
\end{tabular}




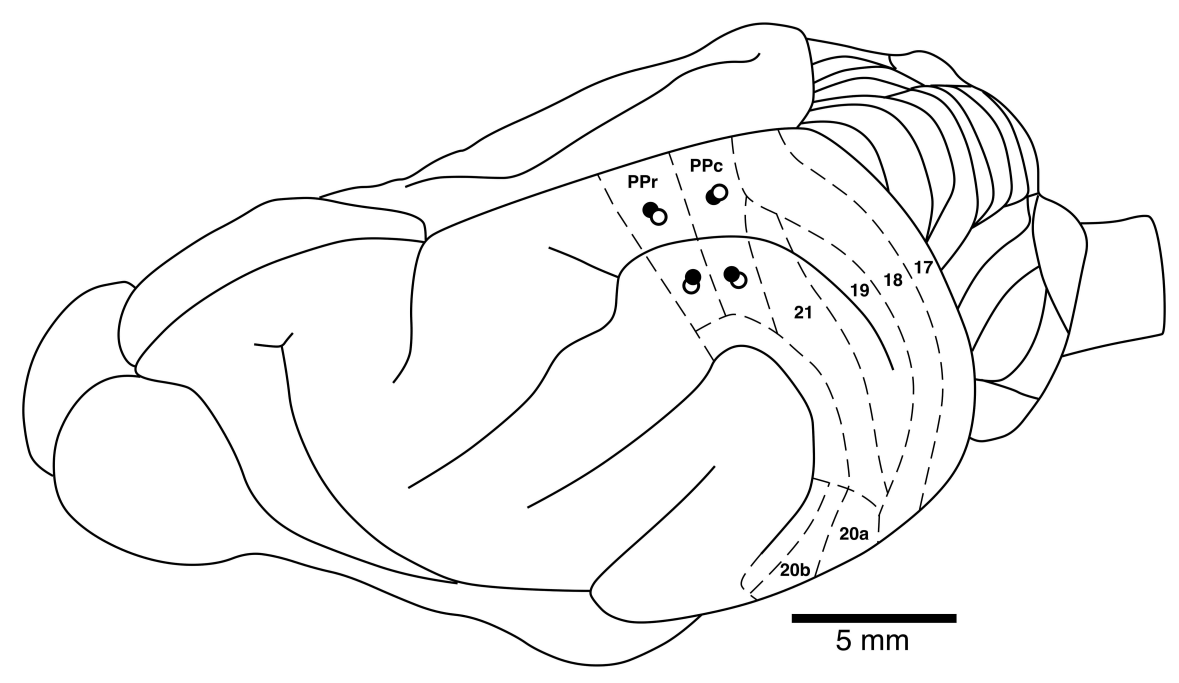

Figure 1: Locations of the injection sites in the parietal cortical areas analyzed in the current study in relation to many of the known boundaries of visual cortical areas in a dorsolateral view of the ferret brain. Closed circles represent the injections sites where the brain was sectioned in a coronal plane. Open circles represent the injections sites where the cerebral cortex was manually semi-flattened for analysis. See list for abbreviations.

$138 \times 80 \mathrm{~mm}(300 \times 300 \mathrm{DPI})$ 

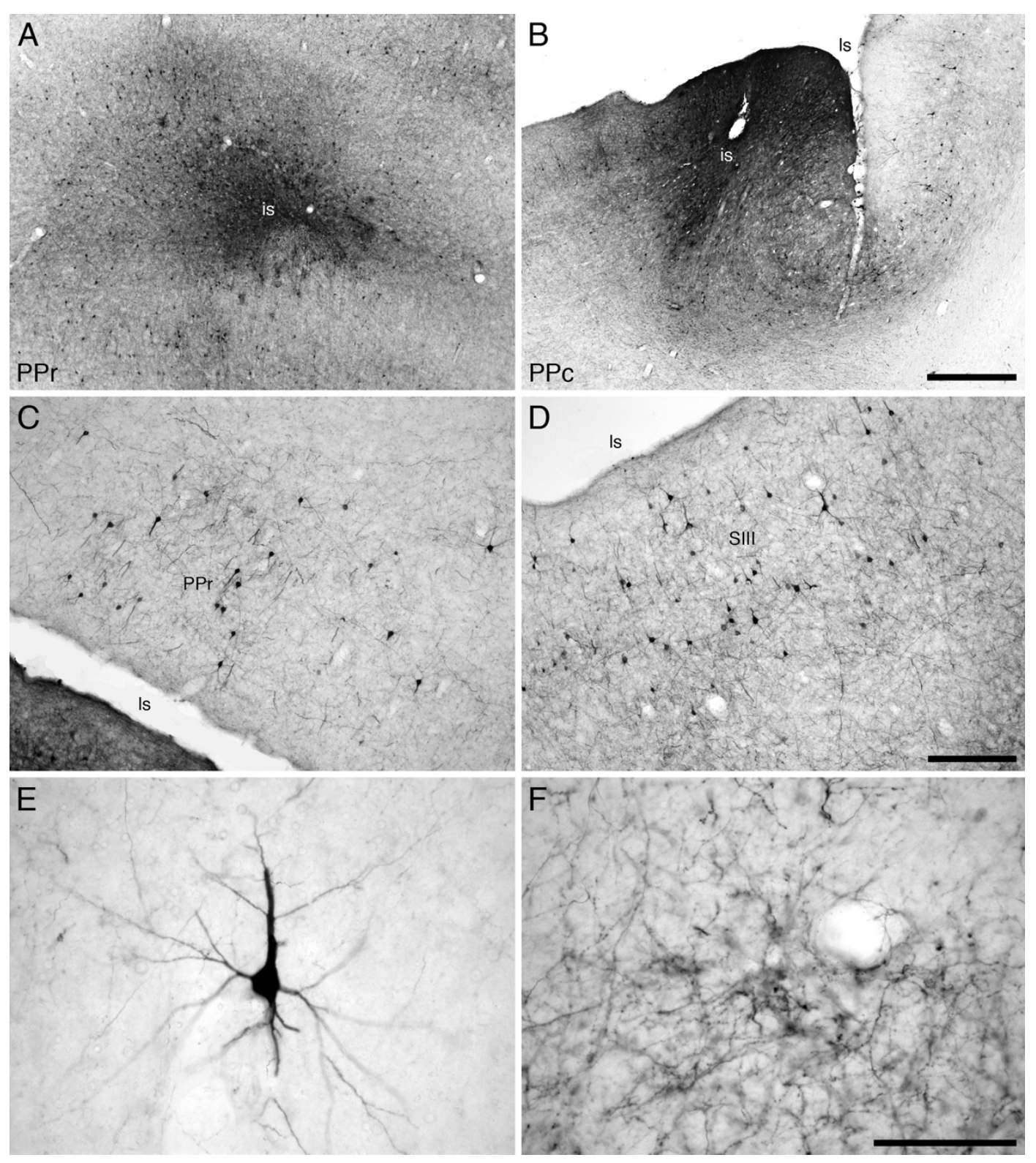

Figure 2: Photomicrographs showing examples of injection sites (a, b) and labeled cells and axons terminals (c-f) that were analyzed in the current study. (a) Injection site (is) in area PPr of a semi-flattened cerebral cortex showing the spread of tracer around the injection site, indicating the areal specificity of the injections made in the current study. (b) Injection site (is) in area PPc from a coronal section through the cerebral cortex, showing the spread of tracer and the limitation of the injection site to the cerebral cortex. Labeled cells and terminals in the medial portion of area PPr (c, medial to the lateral sulcus, Is) and SIII (d, lateral to the lateral sulcus) following transport from an injection site in area PPr. (e) High magnification image of a retrogradely labeled cell in area SII following transport from the injection site depicted in a. (f) High magnification image of anterogradely labeled axons in area 21 following transport from the injection site depicted in $\mathbf{a}$. Scale bar in $\mathbf{b}=500 \mu \mathrm{m}$ and applies to $\mathbf{a}$ and $\mathbf{b}$. Scale bar in $\mathbf{d}=250$ $\mu \mathrm{m}$ and applies to $\mathbf{c}$ and $\mathbf{d}$. Scale bar in $\mathbf{f}=100 \mu \mathrm{m}$ and applies to $\mathbf{e}$ and $\mathbf{f}$. In images $\mathbf{a}, \mathbf{c}, \mathbf{d}$, and $\mathbf{f}$, the midline of the brain is to the top of the image and rostral to the left. In image $\mathbf{b}$, medial is to the right and dorsal to the top. In image e the pial surface has been rotated to the top of the image. 

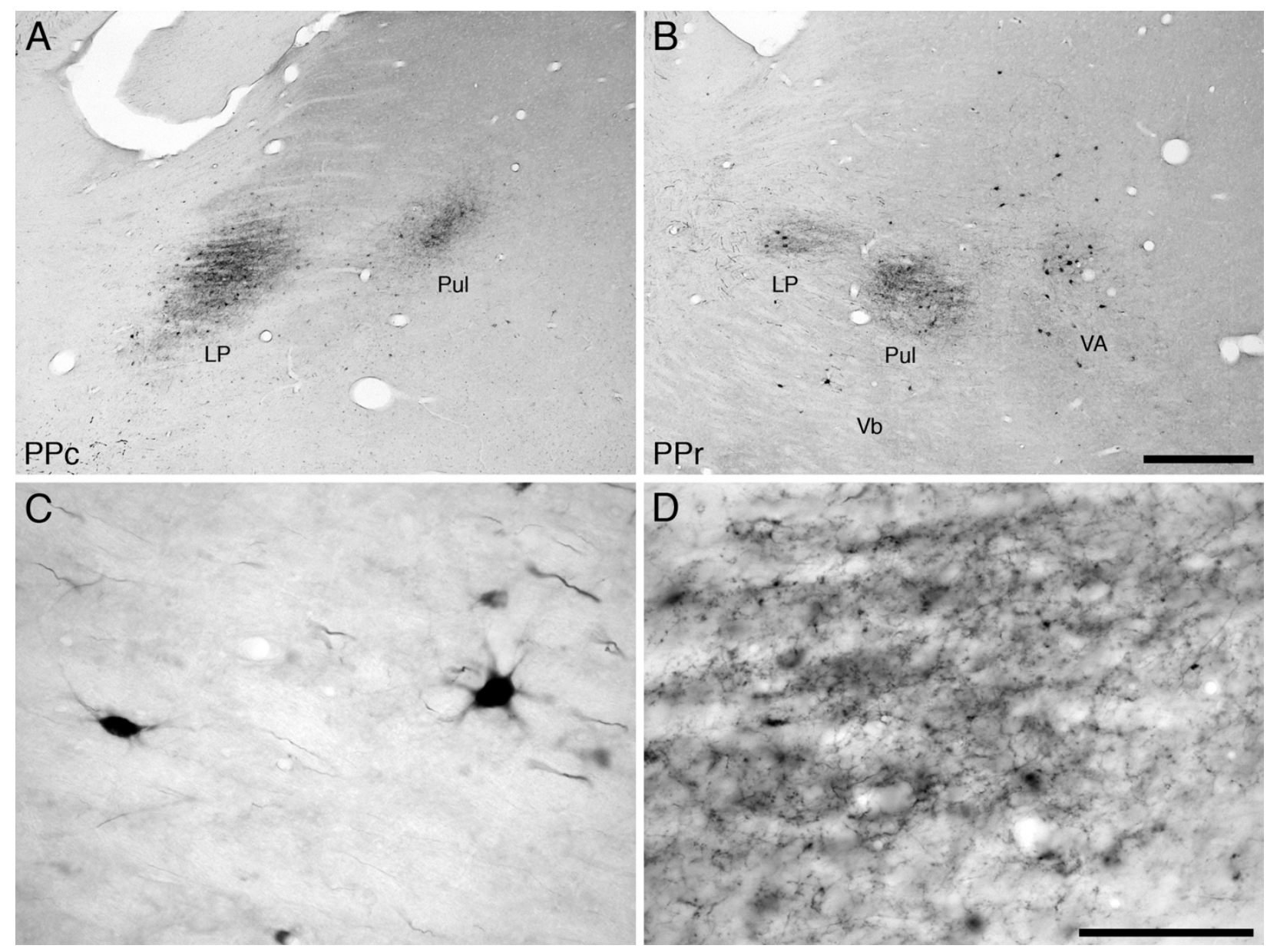

Figure 3: Photomicrographs showing examples of retrogradely labelled cells and anterogradely labelled axons in the visual and motor thalamus of the ferret following injections into the parietal visual areas PPc (a), and PPr (b). (c) High magnification image of retrogradely labelled cells in the ventrobasal complex (Vb) following transport from the injection site in area PPr. (d) High magnification image of anterogradely labelled axons in the pulvinar nucleus following transport from the injection site in area PPc. Scale bar in $\mathbf{b}=500 \mu \mathrm{m}$ and applies to $\mathbf{a}$ and $\mathbf{b}$. Scale bar in $\mathbf{d}$ $=100 \mu \mathrm{m}$ and applies to $\mathbf{c}$ and $\mathbf{d}$. In all images dorsal is to the top and medial to the right. See list for abbreviations.

(300 x 300 DPI) 


\section{Ipsilateral}
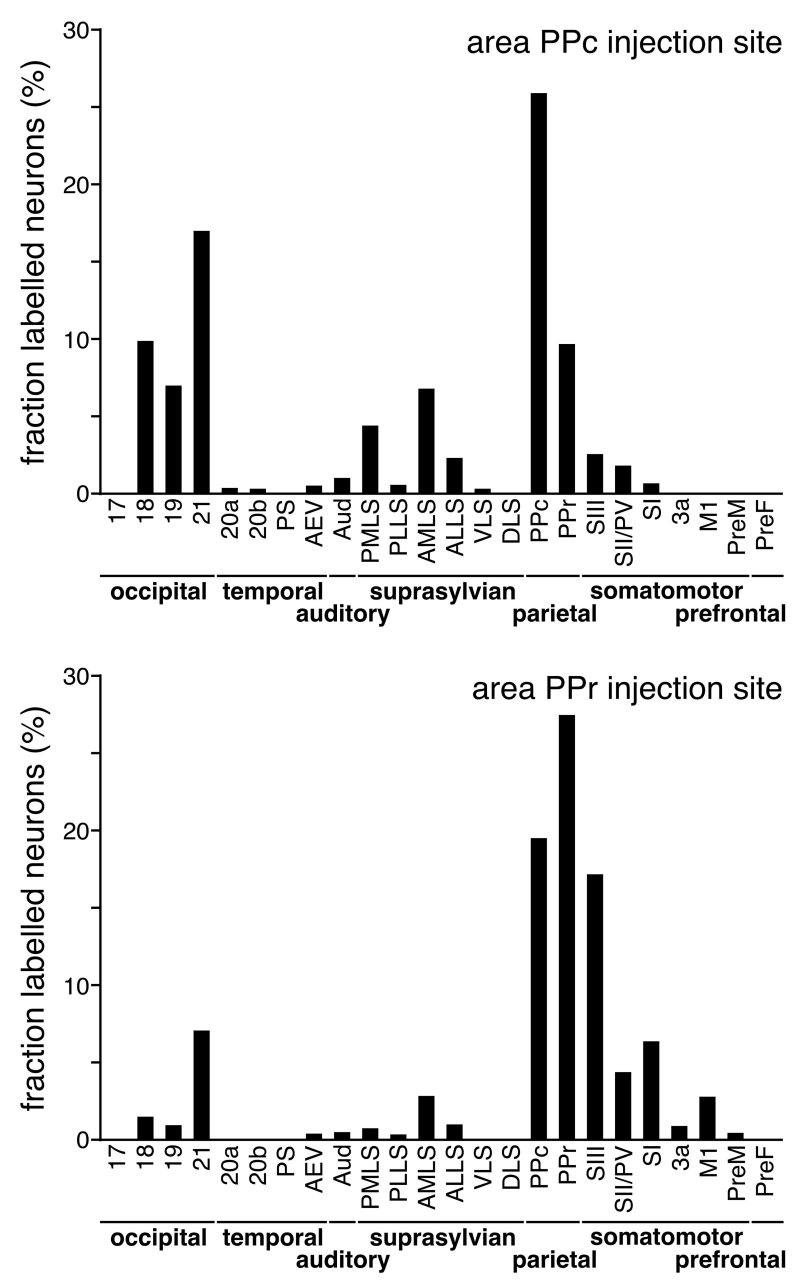

Contralateral
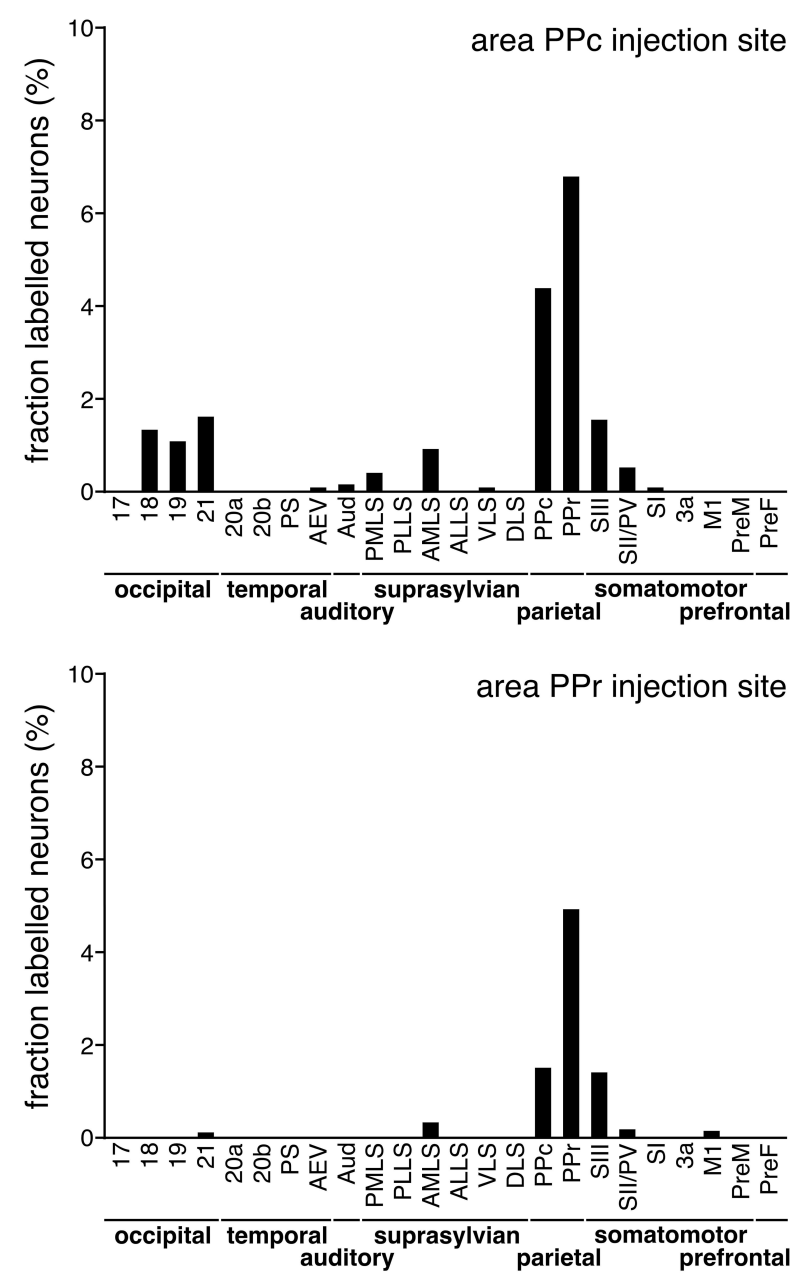

Figure 4: Graphs depicting the quantitative assessment of retrograde connectivity strength within and between cortical areas in the cerebral hemispheres ipsilateral (left column) and contralateral (right column) to the injection sites made in areas PPc (top two graphs) and PPr (bottom two graphs). The values are expressed in percentages, being the fraction of labelled neurons occurring in each cortical area. See list for abbreviations. Note that the majority of retrogradely labelled cells are found within the cortical area injected, and that both parietal areas are connected to each other, both ipsilaterally and contralaterally.

186x157mm (300 x $300 \mathrm{DPI})$ 

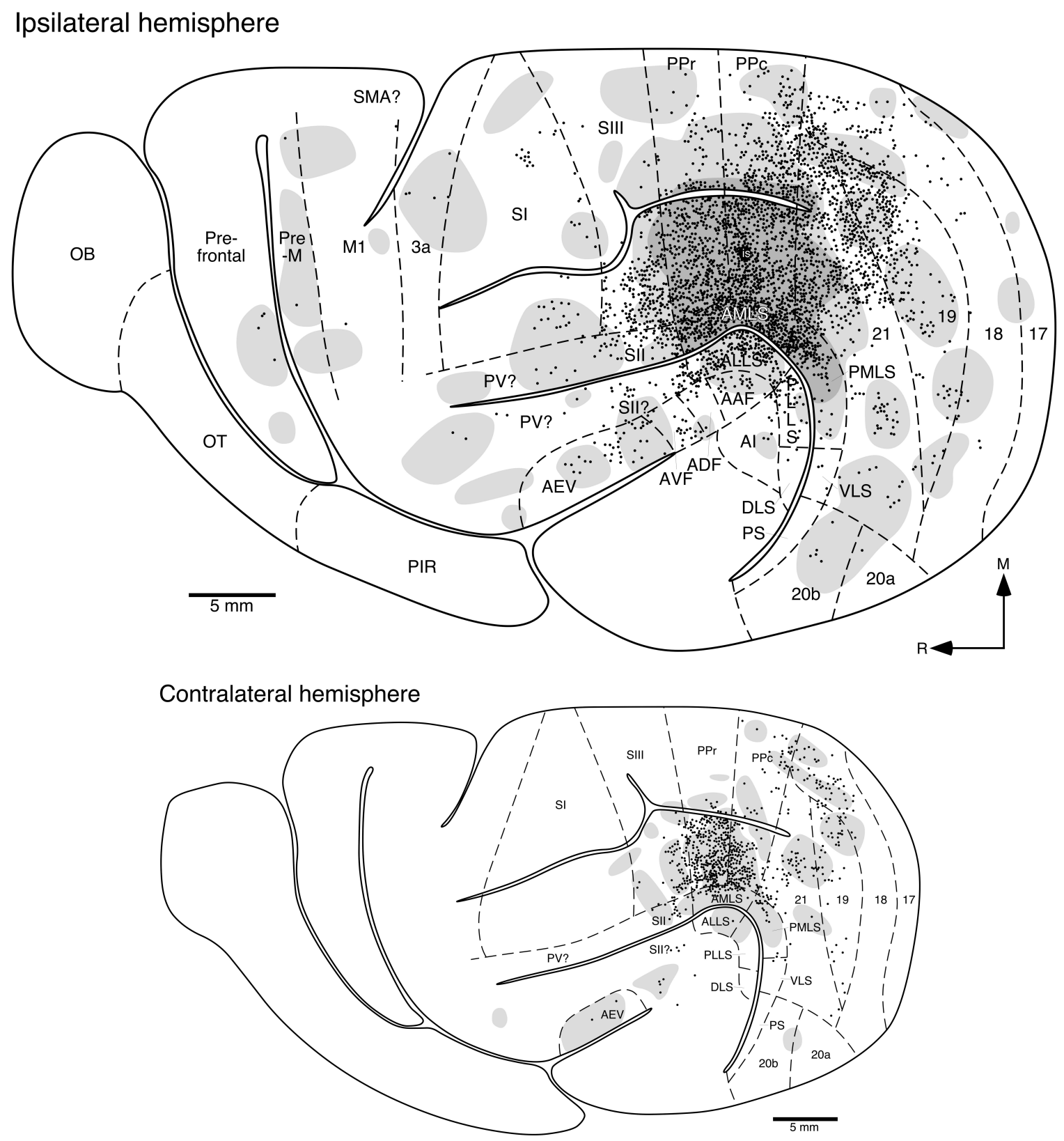

Figure 5: Location of retrogradely labelled cortical neurons (filled circles) and anterogradely labelled axons and axon terminals (dense labelling in the darker grey shading, light labelling in the lighter grey shading) following transport from the injection site (is) located in the lateral portion of area PPc. The upper larger image represent the distribution of cells and axons in the ipsilateral semi-flattened cerebral hemisphere, while the lower smaller image represents the semi-flattened cerebral hemisphere contralateral to the injection site. Note that ipsilaterally, extensive connectivity is seen through the occipital visual areas (19 and 21), the suprasylvian visual areas (AMLS, ALLS and PMLS) as well as the third somatosensory area (SIII). In addition, broad, but weaker, reciprocal connectivity is observed in the temporal visual and auditory regions, the rostral somatosensory regions, motor, pre-motor and prefrontal regions, as well as the claustrocortical regions. The contralateral connectivity is much weaker, and less widespread, but still extensive. Areal boundaries were demarcated using alternative sections stained for cytochrome oxidase and the boundaries represent approximations based on this stain and available maps of the ferret brain (Manger, Kiper, Masiello, Murillo, Tettoni, Hunyadi, \& Innocenti, 2002a; Manger et al., 2002b; Manger, Nakamura, Valentiniene, \& Innocenti, 2004; Manger, Engler, Moll, \& Engel, 2005; Manger, Engler, Moll, \& Engel, 2008; Manger, Restrepo, \& Innocenti, 2010; Bizley, Nodal, Nelken, \& King, 2005; Homman-Ludiye et al., 2010). See list for abbreviations. 

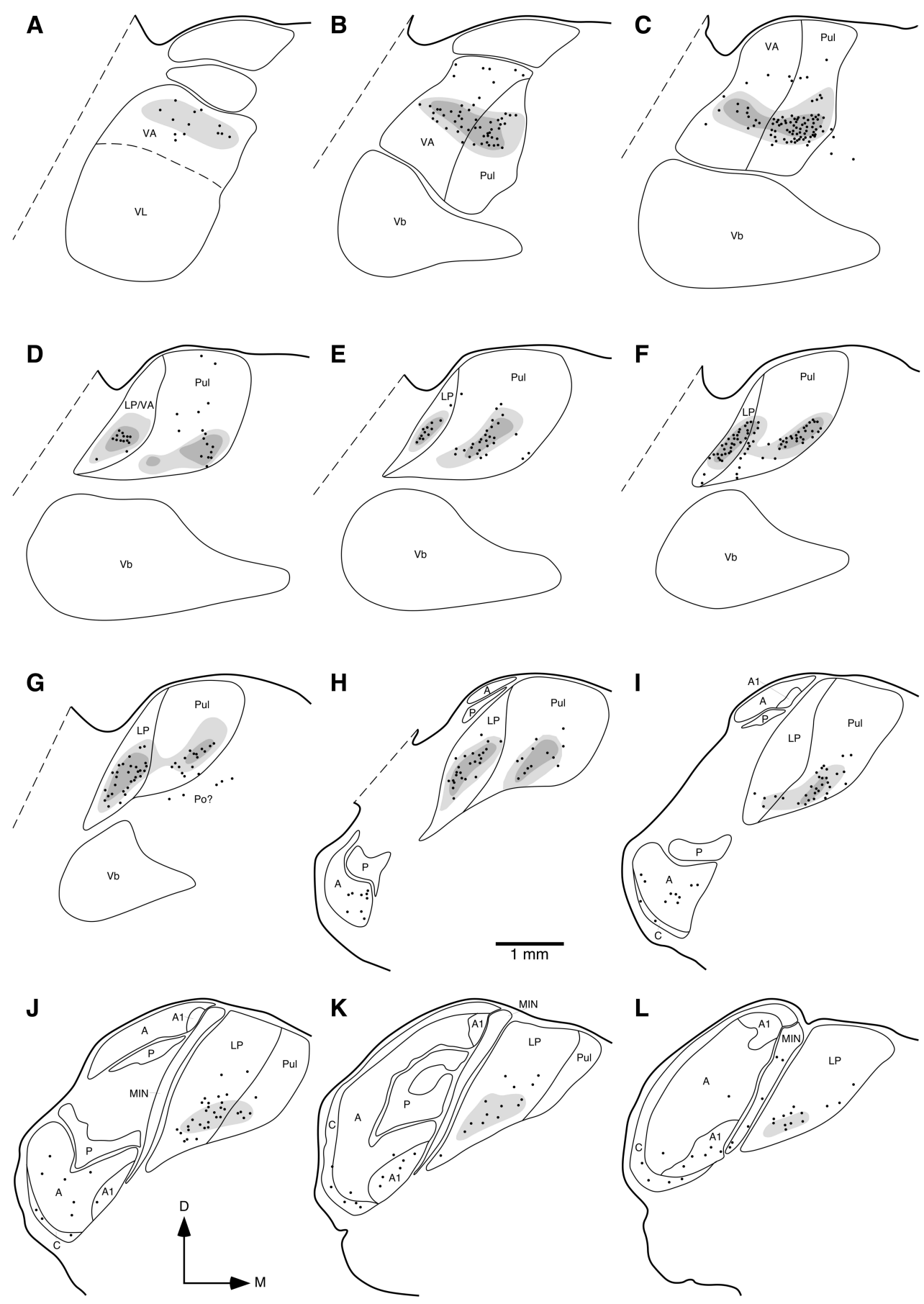

Figure 6: Diagrammatic reconstructions of the location of retrogradely labelled cells (filled circles) and anterogradely labelled axons and axon terminals (dense labelling in the darker grey shading, light labelling in the lighter grey shading) in the visual and motor thalamus of the ferret following injection of tracer into the lateral portion of the parietal visual area PPc. a represents the most rostral coronal section, with I being the most caudal. Each section is approximately $400 \mu \mathrm{m}$ apart. Note the dense reciprocal connectivity with the lateral posterior (LP), pulvinar (Pul) and ventral anterior (VA) nuclei, and limited label in the lateral geniculate nucleus. In all images dorsal (D) is to the top and medial (M) to the right. See list for abbreviations. 

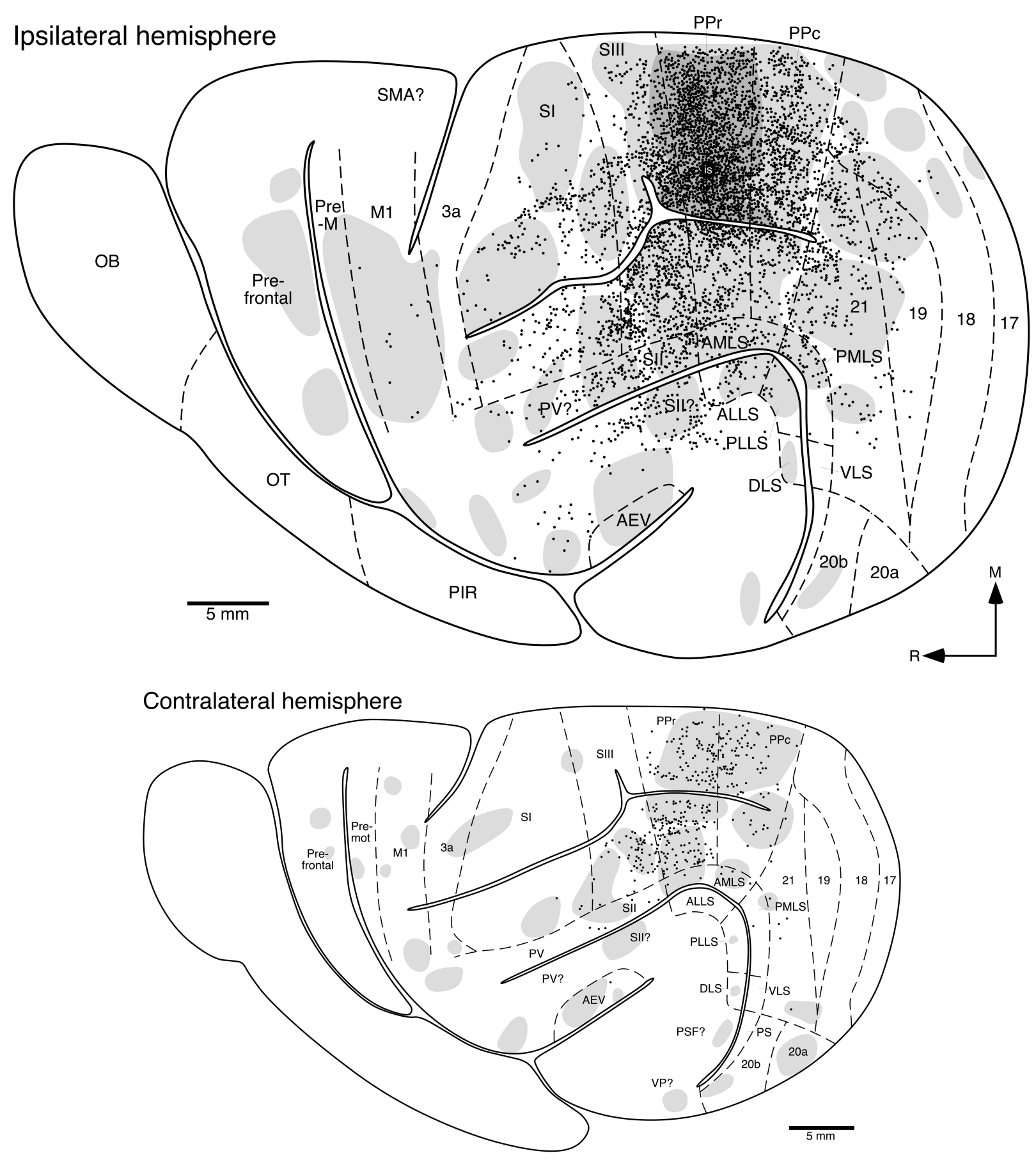

Figure 7: Location of retrogradely labelled cortical neurons (filled circles) and anterogradely labelled axons and axon terminals (dense labelling in the darker grey shading, light labelling in the lighter grey shading) following transport from the injection site (is) located in the medial portion of area PPr. All conventions and abbreviations as provided in the legend to Fig. 5. Note that ipsilaterally, extensive connectivity is seen through the occipital visual areas (19 and 21), the somatosensory areas (SI, SII, SIII and PV?). In addition, broad, but weaker, connectivity is observed in the suprasylvian visual area, the motor, pre-motor and prefrontal regions, as well as the claustrocortical regions. The contralateral connectivity is much weaker, and similarly widespread. See list for abbreviations. 

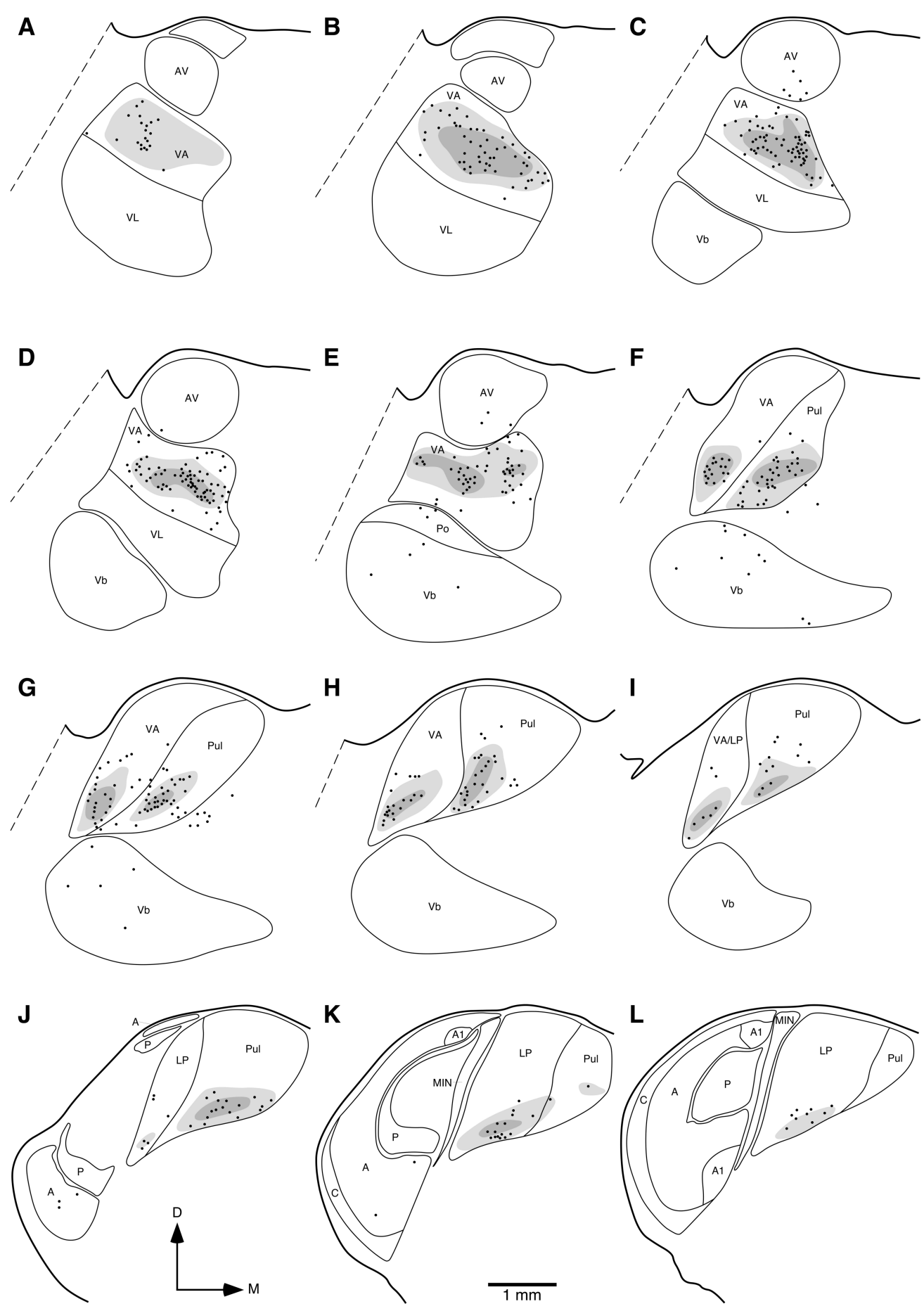

Figure 8: Diagrammatic reconstructions of the location of retrogradely labelled cells (filled circles) and anterogradely labelled axons and axon terminals (dense labelling in the darker grey shading, light labelling in the lighter grey shading) in the visual and motor thalamus of the ferret following injection of tracer into the medial portion of the parietal visual area PPr. a represents the most rostral coronal section, with I being the most caudal. Each section is approximately $400 \mu \mathrm{m}$ apart. Note the dense connectivity with the pulvinar nucleus of the visual thalamus and the ventral anterior (VA) nucleus of the motor thalamus, with some weak connectivity with the lateral geniculate nucleus and the ventrobasal nuclei. Conventions and abbreviations as provided in the legend to Fig. 6 .

$187 \times 266 \mathrm{~mm}(300 \times 300 \mathrm{DPI})$ 


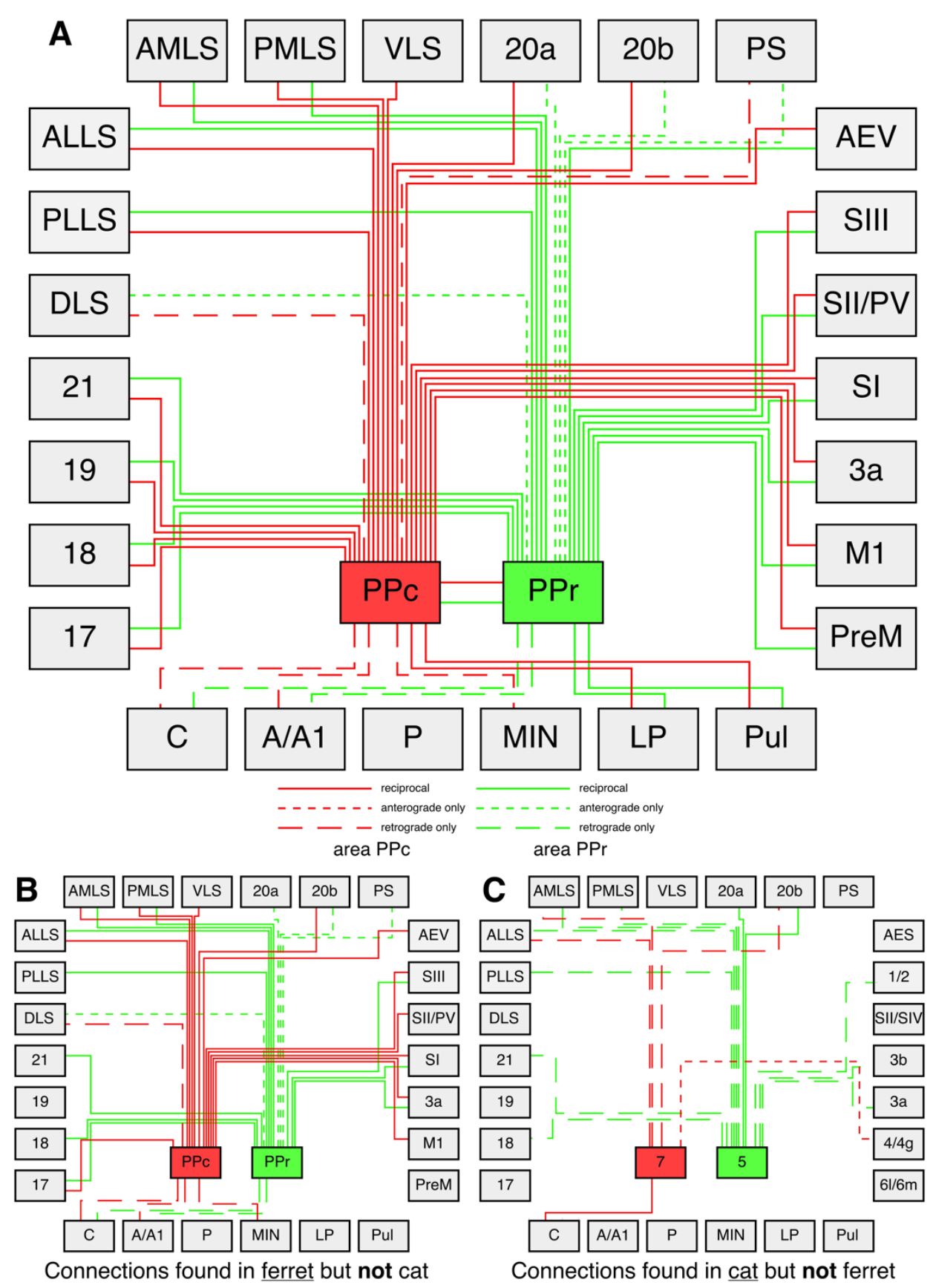

Figure 9: Wiring diagrams depicting the connectivity of areas PPc and PPr with each other, other visual cortical areas ipsilaterally, and the visual thalamus in the ferret (a), the connections of these areas observed in the ferret but not the cat (b), and the connections of these areas overs in the cat but not the ferret (c). Each colour represents a specific cortical area (PPc - red, PPr - green), with solid lines representing reciprocal connections, short dashed lines anterograde connections only, and long dashed lines retrograde connections only. (a) Of the potential 156 connections (for each cortical area 26 potential reciprocal, anterograde only or retrograde only connections) of these two areas to the different visual cortical and thalamic regions, a total of 50 connections are found in the ferret. (b) In the ferret 32 connections not found in the cat were observed, while (c) in the cat 16 connections not found in the ferret were observed (Garol, 1942; Jones \& Powell, 1968; Heath \& Jones, 1971; Kawamura, 1973; Robertson \& Cunningham, 1981; Raczkowski \& Rosenquist, 1983; Babb et al., 1984; Symonds \& Rosenquist, 1984; Avendaño et al., 1988; Scannell et al., 1995, 1999). 